\title{
Culler-Shalen seminorms of fillings of the Whitehead link exterior
}

\author{
Gabriel Indurskis
}

\begin{abstract}
We determine the total Culler-Shalen seminorms for the 3-manifolds $W_{p / q}:=W(p / q, \cdot)$ obtained by Dehn filling with slope $p / q$ on one boundary component of the Whitehead link exterior $W$ when $p$ is odd. As part of the proof, we use an explicit parametrization of the eigenvalue variety of $W$ to find a one-variable polynomial whose roots characterize characters of p-reps of $\pi_{1}\left(W_{p / q}\right)$, i.e. representations with values in $\mathrm{SL}_{2}(\mathbb{C})$ which are parabolic on the peripheral subgroup.
\end{abstract}

\section{Introduction}

Let $W$ be the exterior of the right-handed Whitehead link in $S^{3}$ with the canonical framing $\left(\mu_{0}, \lambda_{0}\right)$ and $\left(\mu_{1}, \lambda_{1}\right)$ as indicated in figure 1. Let $W_{p / q}:=W(p / q, \cdot)$ be the manifold obtained from $W$ by Dehn filling the first boundary torus along the slope $p / q$, i.e. by attaching a solid torus by identifying its meridian with the curve $\mu_{0}^{p} \lambda_{0}^{q}$. Here $p$ and $q$ are coprime integers, and unless otherwise noted we will fix the convention $q>0$. (Note that there is an ambient isotopy taking one component of the link to the other, so in fact $W(p / q, \cdot) \cong W(\cdot, p / q)$. To avoid confusion, we will nevertheless always consider the boundary with curves $\mu_{0}, \lambda_{0}$ to be filled, and the curves $\mu_{1}$ and $\lambda_{1}$ to lie on the remaining boundary torus of $W_{p / q}$ after filling.)

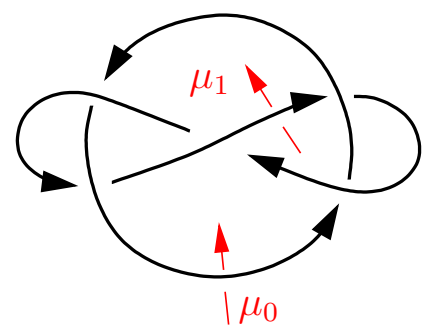

FiguRE 1. The right-handed Whitehead link

1991 Mathematics Subject Classification. Primary 57M27; Secondary 20C15, 57N10, 57R65.

Key words and phrases. 3-manifold, Dehn surgery, character variety, Culler-Shalen seminorm.

This work was supported in part by a scholarship of the Institut des Sciences Mathématiques, Montréal. 
The manifolds $W_{p / q}$ constitute a rich class of 3-manifolds whose boundary consists of one torus, and contain important infinite subfamilies of well-known manifolds such as the twist knot exteriors (for $p / q=1 / n, n \in \mathbb{Z}$ ) and an important class of once-punctured torus bundles (for $p / q=m \in \mathbb{Z}$, cf. [HMW92]). Note that for example the figure- 8 knot complement $(p / q=-1)$ and its so-called sister manifold $(p / q=5)$ are part of this family. Since the Whitehead link exterior admits a complete hyperbolic structure of finite volume, the same is true for all but a finite number of the manifolds $W_{p / q}$ (cf. [Thu79]). Furthermore, unless $p / q \in\{0,4\}$, all manifolds $W_{p / q}$ are small manifolds, i.e. they do not contain any closed essential surfaces (cf. [BGZ01, Appendix]).

The goal of this paper is to determine the total Culler-Shalen seminorm of the filled manifold $W_{p / q}$ when $p$ is odd, in terms of the filling slope $p / q$. This work can be seen as a generalization of similar work previously done for twist knots by Boyer, Mattman and Zhang ([BMZ97]). The results given in this paper completely include these previous results, and extend them to a much larger class of fillings.

TheOREM 1. Let $p$ and $q$ be coprime integers with $q>0$ and $p$ odd, $p / q \neq 3$. The total Culler-Shalen seminorm of the manifold $W_{p / q}=W(p / q, \cdot)$ is given by

$$
\|\gamma\|=\sum_{j=1}^{3} a_{j} \Delta\left(\gamma, \beta_{j}\right),
$$

where $\Delta(\cdot, \cdot)$ denotes the geometric intersection number of two slopes, the slopes $\beta_{j}(j=1,2,3)$ are the possible boundary slopes for $W_{p / q}$ given in table 1 below, and the coefficients $a_{1}, a_{2}$, and $a_{3}$ are given in table 2 , Furthermore, the minimal non-zero total norm $s$ is as given in the last column of table 2 on page 3 .

We remark that the condition that $p$ be odd (which is required in the proofs of section 5 to avoid a possible obstruction to lifting $\mathrm{PSL}_{2}(\mathbb{C})$ representations) automatically excludes the special cases $W_{0}$ and $W_{4}$ (both large, toroidal manifolds which do not admit a complete hyperbolic structure), as well as $W_{6}$ : Filling this manifold a second time with slope 1 produces the closed manifold $L_{2} \# L_{3}$, a connected sum of lens spaces, causing the arguments in section [5 to fail. The same argument applies to $W_{3}$, which therefore has to be excluded separately.

\begin{tabular}{c||c|ccc|c}
$r=p / q \in$ & $\beta_{1}$ & $\beta_{2}$ & & $\beta_{3}$ \\
\hline$[-\infty, 0]$ & 4 & $4 r^{-1}=\frac{4 q}{p}$ & $\in$ & {$[-\infty, 0]$} & 0 \\
{$[0,2]$} & 4 & $4 r^{-1}+2=\frac{2 p+4 q}{p}$ & $\in$ & {$[4, \infty]$} & 0 \\
{$[2,4]$} & 4 & $6-r=\frac{-p+6 q}{q}$ & $\in$ & {$[2,4]$} & 0 \\
{$[4, \infty]$} & 4 & $4(r-2)^{-1}=\frac{4 q}{p-2 q}$ & $\in$ & {$[0,2]$} & 0
\end{tabular}

TABle 1. Possible boundary slopes of $W_{p / q}$ 
CULLER-SHALEN SEMINORMS OF FILLINGS OF THE WHITEHEAD LINK EXTERIOR 3

\begin{tabular}{c||c|c|c|c}
$p / q \in$ & $a_{1}$ & $a_{2}$ & $a_{3}$ & $s$ \\
\hline$(-\infty, 0)$ & $-p+2 q-1$ & 2 & $2 q-2$ & $-3 p+4 q-3$ \\
$(0,2)$ & $-p+2 q-1$ & 2 & $2 q-2$ & $p+4 q-3$ \\
$(2,3) \cup(3,4)$ & $p-2 q-1$ & 4 & $2 q-2$ & $p+4 q-3$ \\
$(4,6) \cup(6, \infty)$ & $p-2 q-1$ & 2 & $2 q-2$ & $3 p-4 q-3$
\end{tabular}

TABLE 2. The parameters for the total Culler-Shalen seminorm of $W_{p / q}$ for $p$ odd, $q>0$

The knowledge of the coefficients $a_{j}$ given in Theorem 1 has immediate consequences: If $a_{j}>0$, the fundamental polygon (the boundary of the norm-ball of radius $s$ ) of the Culler-Shalen seminorm has a vertex corresponding to $\beta_{j}$, and Lemma 6.1 of BZ96 then shows that in this case $\beta_{j}$ is a strongly detected, strict boundary slope, i.e. the slope of an essential surface associated to some ideal point of the character variety of $W_{p / q}$, which is neither a fibre nor semi-fibre. Inspection of table 2 therefore implies:

COROllary 2. Let $p$ and $q$ be coprime integers with $q>0, p$ odd, and $p / q \neq 3$.

(1) $\beta_{1}=4$ is a strongly detected slope of $W_{p / q}$ unless $p=2 q \pm 1$.

(2) $\beta_{2}$ is always a strongly detected slope of $W_{p / q}$.

(3) $\beta_{3}=0$ is a strongly detected slope of $W_{p / q}$ iff $q>1$, otherwise it is the slope of a fibre in the once-punctured torus bundle $W(p)$.

An interesting by-product of the proof of Theorem 1 is the complete determination of the so-called p-reps of the fundamental group of $W_{p / q}$, which are of interest in their own right. These are representations into $\mathrm{SL}_{2}(\mathbb{C})$ which are parabolic on the peripheral subgroup, and were first studied for knot groups by Riley (cf. Ril72]). (To be precise, the generator corresponding to the meridian of the boundary torus is sent to a non-trivial element of trace \pm 2 in $\mathrm{SL}_{2}(\mathbb{C})$. As a consequence, the generator corresponding to the longitude will be either parabolic as well, or $\pm I$.) In his classical work, he showed how under certain conditions on the group presentation (e.g. for the fundamental group of a 2-bridge knot) all such p-reps can be characterized by the complex roots of a single one-variable polynomial. This is in contrast to the general situation, where only a characterization by a system of several polynomial equations can be expected.

Passing through the so-called eigenvalue variety (as defined by Tillmann in Til05]) of the unfilled Whitehead link exterior $W$, we are able to find a similar approach which seems naturally adapted to the situation of Dehn filling to determine a single one-variable polynomial whose roots are in two-to-one correspondence with the set of eigenvalues of p-reps. This in turn is shown to be in bijective correspondence with the set of p-reps itself, and we obtain a complete characterization of all p-reps of $W_{p / q}$ :

THEOREM 3. The conjugacy classes of irreducible p-reps of the manifold $W_{p / q}$ ( $p$ can be odd or even) are in two-to-one correspondence with the non-trivial roots $s \notin\{0, \pm 1\}$ of the Laurent polynomial

$$
\operatorname{res}_{p, q}(s)=s^{p-2 q}+(-1)^{q+1} 2 T_{q}(y)+s^{-p+2 q},
$$

where $y=\frac{1}{2}\left(-s^{2}+4-s^{-2}\right)$, and $T_{q}(y)$ is the q-th Chebyshev polynomial of first type. The conjugacy classes of non-abelian reducible p-reps are in two-to-one correspondence with the non-trivial roots of unity $s \neq \pm 1, s^{p}=1$. 
We remark that whereas it seems a hard analytic problem to determine if the non-trivial roots of these polynomials are always simple, this is in fact a consequence of Theorem 1 when $p$ is odd:

COROLlary 4. When $p$ is odd (including $p / q=3$ ), all non-trivial roots $s \notin$ $\{0, \pm 1\}$ of res $_{p, q}$ are simple.

Proof. Note that even though Theorem 1 excludes $p / q=3$, we can include it here simply by manually inspecting $\operatorname{res}_{3,1}(s)=-s^{3}(s+1)^{2}\left(s^{2}-3 s+1\right)$.

For all other cases with $p$ odd, Theorem 1 shows that the upper bounds for the count of distinct non-trivial roots of $\operatorname{res}_{p, q}$ obtained in section 3.5 are actually attained, which can only happen if these roots are all simple.

We note that experimental evidence seems to suggest that this also holds when $p$ is even. Furthermore, we would like to point out that all our results would immediately extend to the case when $p$ is even if a work-around for the lifting obstruction in section 5 could be found.

The organization of this paper is as follows:

Section 2: After discussing some preliminaries about character varieties and the Culler-Shalen seminorm, we give the general outline for the proof of Theorem 1, We also determine the set of possible boundary slopes of $W_{p / q}$.

Section 3; In this section, we identify the p-reps of the manifold $W_{p / q}$ with points in the eigenvalue variety of the unfilled manifold $W$ characterized by the roots of a single one-variable Laurent polynomial $\operatorname{res}_{p, q}$, proving Theorem 3 . A detailed analysis of the properties and symmetries of these recursively defined polynomials is given, including an upper bound for the number of their roots in terms of $p$ and $q$, which in turn gives an upper bound for the number of conjugacy classes of p-reps.

Section 4: We show that the minimal nonzero value of the Culler-Shalen seminorm of $W_{p / q}$ is precisely equal to the number of conjugacy classes of p-reps, and therefore satisfies the same bounds as shown in the previous section: Using group cohomology calculations and the characterization of p-reps from section 3, it is shown that each character of a p-rep is a smooth point on a non-trivial curve in the character variety of $W_{p / q}$, and that it furthermore is a simple root of the trace function $f_{\mu_{1}}$.

Section 5: We explicitly determine the Culler-Shalen seminorm of slopes $\sigma$ resulting in Seifert-fibered manifolds $W_{p / q}(\sigma)$ when $p$ is odd. This is done by a detailed study of $\mathrm{PSL}_{2}(\mathbb{C})$ representations of the orbifold fundamental groups of their base orbifolds, which can always be lifted to $\mathrm{SL}_{2}(\mathbb{C})$ representations when $p$ is odd, but not necessarily if $p$ was even.

Section 6: The total Culler-Shalen seminorm is determined for $p$ odd: Combining the results from the previous sections, we solve the linear systems relating the seminorms obtained for the Seifert fillings with the coefficients determining the seminorm.

Acknowledgments. The work contained in this paper forms part of the author's dissertation and could not have been achieved without the never-waning support of his supervisor, Steven Boyer, for which the author would therefore like to express his sincerest thanks. Further thanks go to Stephan Tillmann for suggesting the "detour" through the 
eigenvalue variety and for many interesting discussions. Finally, the author would like to thank the eagle-eyed anonymous reviewer for catching a potentially show-stopping sign mistake, and whose comments and recommendations have greatly improved the readability of this paper.

\section{Preliminaries and outline}

We briefly recall the construction of the Culler-Shalen norm developed in CGLS87, and its generalization to a seminorm by Boyer and Zhang in BZ98.

Let $M$ be a connected, orientable, compact, irreducible and boundary-irreducible 3manifold with boundary $\partial M$ consisting of one torus. Unless noted otherwise, we will generally suppress the base point of fundamental groups.

Denote by $\mathfrak{R}(M):=\operatorname{Hom}\left(\pi_{1}(M) ; \mathrm{SL}_{2}(\mathbb{C})\right)$ the representation variety of $M$, and by $\mathfrak{X}(M)$ its character variety, i.e. the set of characters of representations in $\mathfrak{R}(M)$ (cf. CS83). These two sets are in fact affine algebraic varieties and as such can be identified with subsets of $\mathbb{C}^{N}$ (for appropriate $N \in \mathbb{N}$ ), defined by a set of polynomial equations. We will occasionally need to consider representations into $\mathrm{PSL}_{2}(\mathbb{C})$, which we will denote by $\bar{\rho} \in \bar{\Re}(M):=\operatorname{Hom}\left(\pi_{1}(M) ; \operatorname{PSL}_{2}(\mathbb{C})\right)$.

Since $M$ is boundary-irreducible, we can identify $\pi_{1}(\partial M)$ with its image under the natural inclusion $\pi_{1}(\partial M) \rightarrow \pi_{1}(M)$ (defined up to conjugation). Denote the first homology of the boundary with integer coefficients $H_{1}(\partial M ; \mathbb{Z})$ by $L$ and regard it as a lattice in the 2-dimensional real vector space $V=H_{1}(\partial M ; \mathbb{R})$. Using the Hurewicz homomorphism, we can identify $L$ with $\pi_{1}(\partial M)$.

A slope $r$ is the isotopy class of an essential, unoriented, simple closed curve on $\partial M$. It can be identified with a pair of elements $\pm \delta= \pm(p, q) \in L$, or with the rational number $p / q \in \mathbb{Q} \cup\{\infty\}$, where $\infty=1 / 0$ and $-\infty=-1 / 0$ are identified. Let $\alpha(r)$ be either one of $\pm \delta$. We will often slightly abuse notation and interchange freely between these notations; for example writing $M(\delta)$ or $M(p / q)$ instead of $M(r)$ to denote the manifold obtained by Dehn filling $M$ along the slope $r$. A slope is called a boundary slope if it represents the isotopy class of the boundary components (on one component) of $\partial M$ of an essential surface $S \subset M$. A boundary slope is called strict if it is the boundary slope of an essential surface $S$ which is neither a fibre or a semi-fibre in a fibre or semi-fibre bundle structure on $M$. For two elements $\delta_{1}, \delta_{2} \in L$, we denote their geometric intersection number, called their distance, by $\Delta\left(\delta_{1}, \delta_{2}\right)$. If $\delta_{i}=\left(p_{i}, q_{i}\right)$, we have $\Delta\left(\delta_{1}, \delta_{2}\right)=\left|p_{1} q_{2}-p_{2} q_{1}\right|$.

Let $\mathfrak{X}_{0} \subset \mathfrak{X}$ be a curve in the character variety $\mathfrak{X}=\mathfrak{X}(M)$, i.e. an irreducible algebraic component of complex dimension 1 . Call $\mathfrak{X}_{0}$ non-trivial if it contains the character of an irreducible representation.

For $\gamma \in \pi_{1}(M)$, define the regular function $I_{\gamma}: \mathfrak{X}_{0} \rightarrow \mathbb{C}$ by $I_{\gamma}\left(\chi_{\rho}\right)=\chi_{\rho}(\gamma)=$ trace $\rho(\gamma)$. Use this to define the map $f_{\gamma}:=I_{\gamma}^{2}-4$, which is also regular and can hence be pulled back to the smooth projective model (i.e. the unique non-singular projective variety birationally equivalent to the curve $\mathfrak{X}_{0}$, cf. Sha94, Ch. II, $\S 4.5$ and 5.3]) $\widetilde{\mathfrak{X}}_{0}$ of $\mathfrak{X}_{0}$ (we will denote this map also by $f_{\gamma}$ ).

For $\gamma \in L$, define $\|\gamma\|_{\mathfrak{X}_{0}}$ as the degree of the map $f_{\gamma}: \widetilde{\mathfrak{X}}_{0} \rightarrow \mathbb{C P}^{1}$. This can be extended naturally to define a function on the real vector space $V:=H_{1}(\partial M ; \mathbb{R})$ :

$$
\|\cdot\|_{\mathfrak{X}_{0}}: H_{1}(\partial M ; \mathbb{R}) \rightarrow[0 ; \infty)
$$

This function is a seminorm on $H_{1}(\partial M ; \mathbb{R})$, called the Culler-Shalen seminorm associated to the curve $\mathfrak{X}_{0}$ (cf. CGLS87, Proposition 1.1.2] for the case of a norm, and BZ98. Section 5] for the general case). Seen as a seminorm on a 2-dimensional real vector space, $\|\cdot\|_{\mathfrak{X}_{0}}$ is either a norm, a nonzero indefinite seminorm, or identically zero. When $M$ is small, the last possibility cannot occur (cf. BZ98, Proposition 5.5]). Furthermore $\operatorname{dim} \mathfrak{X}(M) \leq 1$ in this case $\left(\mathrm{cf}\right.$. $\left.\mathbf{C C G}^{+} \mathbf{9 4}\right]$ ), and it is therefore natural to consider the sum of the Culler-Shalen seminorms over all non-trivial curves $\mathfrak{X}_{i} \subset \mathfrak{X}(M)$, which we will 
call the total Culler-Shalen seminorm:

$$
\|\gamma\|:=\sum_{i}\|\gamma\|_{i}
$$

We denote the union of all non-trivial curves in $\mathfrak{X}(M)$ by $\mathfrak{X}^{\text {irr }}(M)$.

If $f_{\gamma}$ is non-constant on $\mathfrak{X}_{0}$ for some $\gamma \in L$, define

$$
s_{\mathfrak{X}_{0}}:=\min \left\{\|\gamma\|_{\mathfrak{X}_{0}} \mid \gamma \in L, \gamma \neq 0, f_{\gamma} \text { not constant on } \mathfrak{X}_{0}\right\},
$$

otherwise set $s \mathfrak{X}_{0}:=0$. We call $s \mathfrak{X}_{0}$ the minimal seminorm on $\mathfrak{X}_{0}$, the corresponding value for the total seminorm is the total minimal seminorm, denoted by $s$.

The following lemma enables us to determine the Culler-Shalen seminorm from only a few known values:

LEMma 5 ([BZ96 Lemma 6.2]). If $\beta_{j}$ are the boundary slopes of $M$ and $\mathfrak{X}_{i} \subset \mathfrak{X}(M)$ is a non-trivial curve (i.e. a curve which contains the character of an irreducible representation), then

$$
\|\gamma\|_{\mathfrak{X}_{i}}=\sum_{j} a_{i j} \Delta\left(\gamma, \beta_{j}\right),
$$

where the $a_{i j}$ are non-negative, even integers independent of $\gamma \in \pi_{1}(M)$.

For the total seminorm, we therefore get

$$
\|\gamma\|=\sum_{j} a_{j} \Delta\left(\gamma, \beta_{j}\right)
$$

with non-negative even integers $a_{j}$.

To determine the total seminorm for the manifolds $W_{p / q}$, it therefore suffices to determine the boundary slopes $\beta_{j}$ and the corresponding coefficients $a_{j}$. Following a technique due to Mattman (cf. Mat02 ), we will use explicit calculations of the seminorm of certain slopes (cf. section 5) together with equation (4) to set up a system of linear equations with unknowns $a_{j}$ and $s$. In our case, this system turns out to be underdetermined, requiring one more piece of information to successfully solve it. We therefore first study the set of p-reps of $W_{p / q}$ (cf. section 3) and use this knowledge to explicitly determine the minimal total seminorm $s$ (cf. section 4). With this information, we are then able to solve the linear systems in section [6] completing the proof of Theorem 1.

We finish this section by determining the set of possible boundary slopes of $W_{p / q}$ :

2.1. Boundary slopes of the unfilled Whitehead link exterior. In HS07, Hoste and Shanahan give an explicit algorithm, building on work of Floyd and Hatcher (cf. FH88) and on the unpublished dissertation of Lash (cf. Las93), to determine the boundary slopes of 2-bridge links. In particular, they show that up to symmetry between the two boundary tori, the left-handed Whitehead link ( $L_{3 / 8}$ in 2-bridge notation) has boundary slope pairs $(0,0),(0, \varnothing),(-4,-2),(-4, \varnothing)$ (here $\varnothing$ signifies that the intersection with one of the boundary components is empty) and the continuous spectrum of slope pairs $\left(2 t^{-1}, 2 t\right),\left(-2 t^{-1},-2-2 t\right),(-3+s,-3-s)$ parametrized by $t \in(\mathbb{Q} \cup\{\infty\}) \cap[0, \infty]$ and $s \in \mathbb{Q} \cap[-1,1]$ (cf. table 4 in [HS07, using $k=1$ ).

After applying an orientation-reversing homeomorphism to switch to the right-handed Whitehead link (which simultaneously changes the signs of all slopes), we use the symmetry between the two boundary components and reparametrize the slope pairs such that the first is always equal to $r=p / q$. The results are shown in table 3 .

2.2. Boundary slopes of the once-filled Whitehead link exterior. Denote the two boundary components of $W$ by $T_{0}$ and $T_{1}$. We now proceed to determine the boundary slopes of the once-filled Whitehead link exterior $W_{p / q}=W(p / q, \cdot)=W \cup_{\mu_{0}^{p} \lambda_{0}^{q}} V$, where $V$ is the attached solid torus. Note that we can view $W$ as lying inside $W_{p / q}$. 


\begin{tabular}{c|ccc}
$r=p / q$ on $T_{0}$ & $p^{\prime} / q^{\prime}$ on $T_{1}$ & & \\
\hline \hline 0 & 0 or $\varnothing$ & & \\
\hline & 2 or $\varnothing$ & & \\
\hline$\varnothing$ & 0 & & \\
\hline 2 or $\varnothing$ & $4 r^{-1}=\frac{4 q}{p}$ & $\in$ & {$[-\infty, 0]$} \\
$r \in[-\infty, 0]$ & $4 r^{-1}+2=\frac{2 p+4 q}{p}$ & $\in$ & {$[4, \infty]$} \\
$r \in[0,2]$ & $6-r=\frac{-p+6 q}{q}$ & $\in$ & {$[2,4]$} \\
$r \in[2,4]$ & $4(r-2)^{-1}=\frac{4 q}{p-2 q}$ & $\in$ & {$[0,2]$}
\end{tabular}

TABLE 3. Boundary slope pairs of the right-handed Whitehead link $W$

Lemma 6. Let $S$ be an essential surface in $W_{p / q}$. Then either $S$ is in fact an essential surface in $W \subset W_{p / q}$, or it is the cap-off of an essential surface $S^{\prime}$ in $W \subset W_{p / q}$. In particular, $S$ has non-empty intersection with at least one of $T_{0}$ and $T_{1}$.

Proof. Let $S$ be an essential surface in $W_{p / q}$. We can isotope $S$ so it transversely intersects $T_{0} \subset W \subset W_{p / q}$ and $T_{1} \subset W_{p / q}$, as well as the core of the filling torus $V$. If $S \cap T_{0}=S \cap T_{1}=\varnothing, S$ can be viewed as a closed essential surface in $W$, which is a contradiction, since $W$ is small. We therefore see that $S$ has to intersect at least one of $T_{0}$ or $T_{1}$.

We now have two cases to consider:

$S \cap \operatorname{int} V=\varnothing$ : In this case, $S$ is in fact an essential surface in $W \subset W_{p / q}$ with empty intersection with $T_{0}$. According to table 3 it therefore must have boundary slope on $T_{1}$ equal to either 0 or 4 .

$S \cap$ int $V \neq \varnothing$ : In this case, the intersection $S \cap T_{0}$ must consist of simple closed curves of slope $p / q$. This implies that $S^{\prime}=S \cap W$ is an essential surface in $W$ with boundary slope $p / q$ on $T_{0}$. Unless $p / q \in\{0,4\}$, this implies that $S^{\prime} \cap T_{1} \neq \varnothing$, and we get the corresponding boundary slope on $T_{1}$ from table 3 . dependent on $p / q$. We remark that if $p / q \in\{0,4\}, S$ could in fact be a closed essential torus in $W_{p / q}$.

We therefore have at most three candidates for boundary slopes in the manifold $W_{p / q}$, as summarized in table 1 on page 2. We have two fixed slopes $\beta_{1}=4$ and $\beta_{3}=0$, and one varying slope $\beta_{2}$ which depends on $p$ and $q$. The parametrization of the latter depends on the range of the filling slope $p / q$.

Note that we have at this point not determined if these slopes are in fact boundary slopes of $W_{p / q}$. For the following, we will rather only assume that the set of boundary slopes of $W_{p / q}$ is contained in the set $\left\{\beta_{1}, \beta_{2}, \beta_{3}\right\}$. The proof of Theorem 1 will then in turn imply Corollary 2, showing that unless $p=2 q \pm 1$ or $q=1$, all three slopes are indeed strongly detected, strict boundary slopes.

\section{The set of p-reps of a once-filled Whitehead link exterior}

The goal of this section is to prove Theorem 3 (in sections 3.1 3.4), as well as to provide upper bounds for the number of non-trivial roots of the Laurent polynomials 
$\operatorname{res}_{p, q}$ (in section 3.5), which in turn then gives upper bounds for the number of conjugacy classes of p-reps (in section 3.6).

The fundamental group of the Whitehead link exterior admits the presentation

which then gives the following presentation for the fundamental group of the filled manifold:

$$
\begin{aligned}
\pi_{1}\left(W_{p / q}\right)=\left\langle\mu_{0}, \mu_{1}\right| \mu_{0} \mu_{1} \mu_{0}^{-1} \mu_{1}^{-1} \mu_{0}^{-1} \mu_{1} \mu_{0} \mu_{1} & =\mu_{1} \mu_{0} \mu_{1} \mu_{0}^{-1} \mu_{1}^{-1} \mu_{0}^{-1} \mu_{1} \mu_{0}, \\
\mu_{0}^{p} \lambda_{0}^{q} & =1\rangle,
\end{aligned}
$$

where

$$
\lambda_{0}=\mu_{0} \mu_{1} \mu_{0} \mu_{1}^{-1} \mu_{0}^{-1} \mu_{1}^{-1} \mu_{0} \mu_{1} \mu_{0}^{-2}=\mu_{1} \mu_{0} \mu_{1}^{-1} \mu_{0}^{-1} \mu_{1}^{-1} \mu_{0} \mu_{1} \mu_{0}^{-1}
$$

(since it commutes with $\mu_{0}$ ) and

$$
\lambda_{1}=\mu_{0} \mu_{1} \mu_{0}^{-1} \mu_{1}^{-1} \mu_{0}^{-1} \mu_{1} \mu_{0} \mu_{1}^{-1} .
$$

Following [Til02, section 5.1.9] (see also Til19, section 5.6] in these proceedings), we first describe a convenient subset of the representation variety $\mathfrak{R}(W)$ : Let $\rho \in \mathfrak{R}(W)$ be a non-abelian representation. Then $\rho$ can be conjugated to be of the following normal form:

$$
\rho\left(\mu_{0}\right)=\left(\begin{array}{cc}
s & c \\
0 & s^{-1}
\end{array}\right) \quad \text { and } \quad \rho\left(\mu_{1}\right)=\left(\begin{array}{cc}
u & 0 \\
1 & u^{-1}
\end{array}\right)
$$

for $s, u \in \mathbb{C}^{*}$ and $c \in \mathbb{C}$ (note that $c=0$ when $\rho$ is non-abelian reducible). We will denote the subset of representations in $\mathfrak{R}\left(W_{p / q}\right)$ of this form by $\mathfrak{C}\left(W_{p / q}\right)$.

Applying (9) to the left-hand side $w_{1}$ and right-hand side $w_{2}$ of the defining relation in (5) yields

$$
0=\rho\left(w_{1}\right)-\rho\left(w_{2}\right)=\left(\begin{array}{cc}
-c f & c\left(u-u^{-1}\right) f \\
\left(s-s^{-1}\right) f & c f
\end{array}\right)
$$

where

$$
\begin{gathered}
f(s, u, c)=\left(s-s^{-1}\right)\left(u-u^{-1}\right)+c\left(s^{-2} u^{-2}-u^{-2}-s^{-2}+4-s^{2}-u^{2}+s^{2} u^{2}\right) \\
+c^{2}\left(2 s^{-1} u^{-1}-s u^{-1}-s^{-1} u+2 s u\right)+c^{3} .
\end{gathered}
$$

This shows that $\mathfrak{C}(W) \subset \mathbb{C}^{3}$ is defined by the equation $f(s, u, c)=0$.

Let now $\rho \in \mathfrak{R}\left(W_{p / q}\right)$ be a representation which sends the meridian $\mu_{1}$ to a parabolic element (a matrix $A \neq \pm I$ with $|\operatorname{trace} A|=2$ ), and which has non-abelian image in $\mathrm{SL}_{2}(\mathbb{C})$. Since $\mu_{1}$ and $\lambda_{1}$ commute, this implies that $\rho\left(\lambda_{1}\right)$ is either $\pm I$ or also parabolic. Following Riley (cf. Ril72 and [Ril75]) we call such a representation an $\mathrm{SL}_{2}(\mathbb{C}) p$ representation, or, for short, $p$-rep. (We remark that Riley actually used the name p-rep for $\mathrm{PSL}_{2}(\mathbb{C})$ representations only, whereas a $\mathrm{SL}_{2}(\mathbb{C})$ representation which projects to a $\mathrm{PSL}_{2}(\mathbb{C})$ p-rep was called sl-rep, c.f. Ril75.)

Denote by $\mathfrak{P} \subset \mathfrak{C}\left(W_{p / q}\right)$ the subset of p-reps of $\pi_{1}\left(W_{p / q}\right)$ of the form (9). Note that by construction, $\mathfrak{C}\left(W_{p / q}\right)$ projects generically 4-to-1 to $\mathfrak{X}\left(W_{p / q}\right)$, corresponding to the action of the Kleinian four-group (generated here by the inversions $(s, u, c) \mapsto\left(s^{-1}, u^{-1}, c\right)$ and $\left.(s, u, c) \mapsto\left(s, u^{-1}, c+\left(s-s^{-1}\right)\left(u-u^{-1}\right)\right)\right)$ on the set of possible bases for the normal form (9). However, for a p-rep $\rho \in \mathfrak{C}\left(W_{p / q}\right)$, we have $u^{2}=1$ (and $s^{2} \neq 1$ ) and hence the projection is 2-to-1. Each conjugacy class of p-reps has therefore precisely two representatives in $\mathfrak{P}$.

Since $\mathfrak{R}\left(W_{p / q}\right) \subset \mathfrak{R}(W)$, each p-rep $\rho \in \mathfrak{R}\left(W_{p / q}\right)$ induces a non-abelian representation $\rho^{\prime} \in \mathfrak{R}(W)$ satisfying $\rho^{\prime}\left(\mu_{0}^{p} \lambda_{0}^{q}\right)=I$ which still sends $\mu_{1}$ to a parabolic element. We will call such a representation $\rho^{\prime}$ a partial p-rep. Conversely, each partial p-rep $\rho^{\prime}$ factors through $\pi_{1}\left(W_{p / q}\right)$, and we therefore have a bijection between the set $\mathfrak{P} \subset \mathfrak{C}\left(W_{p / q}\right)$ of p-reps in normal form (9) and the set $\mathfrak{P}^{\prime} \subset \mathfrak{C}(W)$ of partial p-reps in normal form. 
Instead of trying to translate the filling relation $\mu_{0}^{p} \lambda_{0}^{q}=1$ into defining equations for the parameters $s, c$ and $u$ in $\mathfrak{C}\left(W_{p / q}\right)$ (which is difficult for general $p$ and $q$ ), we will now restrict our attention to the eigenvalues of p-reps.

3.1. Detour through the eigenvalue variety. The eigenvalue variety of a manifold whose boundary consists of a finite number of tori was introduced by Tillmann in Til05 as a generalization of the $A$-polynomial for knots, and an explicit parametrization of the eigenvalue variety of the Whitehead link exterior can be found in his thesis (cf. Til02 section 5.4.3]) and in Til19, Appendix B.1] in these proceedings.

Let $\rho \in \mathfrak{C}(W)$. For $\gamma \in \pi_{1}(W)$, let

$$
\begin{aligned}
\theta_{\gamma}: & \mathfrak{C}(W) \rightarrow \mathbb{C} \\
\rho & \mapsto \rho(\gamma)_{1,1}
\end{aligned}
$$

be the map taking $\rho$ to the upper left entry of the image $\rho(\gamma)$. Now define the eigenvalue map to be

$$
\begin{aligned}
e: & \mathfrak{C}(W) \rightarrow \mathbb{C}^{4} \\
& \rho \mapsto\left(\theta_{\mu_{0}}(\rho), \theta_{\lambda_{0}}(\rho), \theta_{\mu_{1}}(\rho), \theta_{\lambda_{1}}(\rho)\right) .
\end{aligned}
$$

Note that this is well-defined, as each $\rho \in \mathfrak{C}(W)$ is triangular on the peripheral subgroup.

The image $e(\mathfrak{C}(W)) \subset \mathbb{C}^{4}$ is a subset of the eigenvalue variety which we will denote by $\mathfrak{E}_{0}(W)$. Tillmann showed in Til02 that the map $e$ has degree one, and that furthermore:

Lemma 7 (Lemma 5.6 in Til02] or Lemma 17 in Til19]). The varieties $\mathfrak{C}(W)$ and $\mathfrak{E}_{0}(W)$ are birationally equivalent.

In particular, the map $e$ has the following inverse map for $s \neq \pm 1$ and $u^{2} \neq s^{2}$ :

$$
e^{-1}: \mathfrak{E}_{0}(W) \rightarrow \mathfrak{C}(W), \quad(s, t, u, v) \mapsto\left(s, u, \frac{s^{2}(t-1)+u^{2}(1-v)}{s^{-1} u^{-1}\left(s^{2}-u^{2}\right)}\right)
$$

As shown in Til02], one can in fact find a pair of inverse maps which together are defined on all but eight points of $\mathfrak{E}_{0}(W)$ :

$$
\begin{array}{rlrl}
e_{1}^{-1}: \mathfrak{E}_{0}(W) & \rightarrow \mathfrak{C}(W), & (s, t, u, v) & \mapsto\left(s, u, \frac{\left(s^{2}-v\right)\left(1-u^{2}\right)}{s u(1+v)}\right) \\
e_{2}^{-1}: \mathfrak{E}_{0}(W) \rightarrow \mathfrak{C}(W), & (s, t, u, v) & \mapsto\left(s, u, \frac{\left(u^{2}-t\right)\left(1-s^{2}\right)}{s u(1+t)}\right)
\end{array}
$$

Four of the points which are not defined by these two maps correspond to the discrete faithful representations of $\pi_{1}(W)$ associated to the complete hyperbolic structure of $W$, parametrized by $t=v=-1$ and $s^{2}=u^{2}=1$. The remaining four points are given by $t=v=-1$ and $s^{2}=u^{2}=-1$.

Since a partial p-rep $\rho^{\prime} \in \mathfrak{C}(W)$ satisfies $u^{2}=1$ and $s^{2} \neq 1$, we see that the eigenvalue map $e$ sends the set $\mathfrak{P}^{\prime}$ bijectively onto a set $\mathfrak{P}^{\prime \prime} \subset \mathfrak{E}_{0}$, as shown in the following diagram:

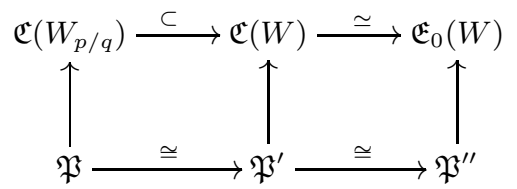

We therefore have a bijective correspondence between the set $\mathfrak{P}$ of $\mathrm{p}$-reps in normal form and a set $\mathfrak{P}^{\prime \prime}$ of eigenvalue tuples in $\mathfrak{E}_{0}$. Since each p-rep $\rho \in \mathfrak{C}\left(W_{p / q}\right)$ and each partial p-rep $\rho^{\prime} \in \mathfrak{C}(W)$ satisfies $\rho\left(\mu_{0}^{p} \lambda_{0}^{q}\right)=I$, we obtain that the set $\mathfrak{P}^{\prime \prime}$ lies in the intersection $\mathfrak{F}$ of the following subvarieties of $\mathfrak{E}_{0}(W)$ :

$$
\mathfrak{P}^{\prime \prime} \subsetneq \mathfrak{F}:=\mathfrak{E}_{0}(W) \cap\left\{s^{p} t^{q}=1\right\} \cap\left\{u^{2}=1\right\} .
$$


It is important to note that $\mathfrak{P}^{\prime \prime}$ is a proper subset of $\mathfrak{F}$, as there are indeed points in $\mathfrak{E}_{0}(W) \cap\left\{s^{p} t^{q}=1\right\}$ corresponding to representations which do not factor through $\pi_{1}\left(W_{p / q}\right)$ (we will see that these are precisely points which satisfy $s^{2}=1$ ).

In summary, we have reduced the generally hard problem of determining the set $\mathfrak{P}$ to the problem of determining the set $\mathfrak{P}^{\prime \prime}$. This task is made feasible by the fact that we can first intersect $\mathfrak{E}_{0}(W)$ with the set $\left\{u^{2}=1\right\}$ (which will significantly simplify the defining polynomials involved), and then intersect the resulting set with the subset $\left\{s^{p} t^{q}=1\right\}$ corresponding to the Dehn filling.

3.2. The subset of the eigenvalue variety corresponding to p-reps. Using the defining polynomial (11) of $\mathfrak{C}(W)$ and using elementary elimination theory, one finds that $e(\mathfrak{C}(W))=\mathfrak{E}_{0}(W) \subset \mathbb{C}^{4}$ can be defined as an algebraic set by the following three polynomials (cf. Til02 section 5.4.3] or Til19 Appendix B.1]):

$$
\begin{aligned}
h_{1}= & t-s^{2} t+s^{2} t^{2}-s^{4} t^{2}-u^{2}-2 s^{2} t u^{2}+s^{4} t u^{2}-t^{2} u^{2}+2 s^{2} t^{2} u^{2} \\
& +s^{4} t^{3} u^{2}+t u^{4}-s^{2} t u^{4}+s^{2} t^{2} u^{4}-s^{4} t^{2} u^{4}, \\
h_{2}= & s^{2}-v-s^{4} v+u^{2} v+2 s^{2} u^{2} v+s^{4} u^{2} v-s^{2} u^{4} v+s^{2} v^{2}-u^{2} v^{2} \\
& -2 s^{2} u^{2} v^{2}-s^{4} u^{2} v^{2}+u^{4} v^{2}+s^{4} u^{4} v^{2}-s^{2} u^{4} v^{3}, \\
h_{3}= & s^{4} t-s^{6} t-s^{2} t u^{2}+s^{4} t u^{2}+s^{6} t^{2} u^{2}-s^{2} u^{2} v \\
& +u^{4} v+s^{2} u^{4} v-2 s^{4} t u^{4} v-u^{6} v+s^{2} u^{6} v^{2} .
\end{aligned}
$$

Intersecting with the subvariety given by $\left\{u^{2}=1\right\}$ then simplifies the defining polynomials to

$$
\begin{aligned}
& g_{1}=(t-1)\left[t(t-1) s^{4}+4 t s^{2}+(1-t)\right], \\
& g_{2}=s^{2}(1-v)(v+1)^{2}, \\
& g_{3}=s^{2}\left[t(t-1) s^{4}+2 t(1-v) s^{2}+\left(v^{2}-t\right)\right] .
\end{aligned}
$$

Since $s \neq 0$, it can be easily verified that the subvariety defined by these polynomials splits into the following two irreducible components:

$$
\begin{gathered}
\mathfrak{E}_{0}(W) \cap\left\{u^{2}=1\right\}= \\
\left\{u^{2}=1, t=v=1\right\} \cup\left\{u^{2}=1, v=-1, s^{4} t^{2}+\left(-s^{4}+4 s^{2}-1\right) t+1=0\right\}
\end{gathered}
$$

Note that points in the first component correspond to reducible representations.

Intersecting this set further, we obtain the set $\mathfrak{F}=\mathfrak{E}_{0}(W) \cap\left\{u^{2}=1\right\} \cap\left\{s^{p} t^{q}=1\right\}$ as

$$
\begin{aligned}
\mathfrak{F}=\mathfrak{F}^{r} \cup \mathfrak{F}^{i}= & \left\{u^{2}=t=v=1, s^{p}=1\right\} \\
& \cup\left\{u^{2}=1, v=-1, s^{4} t^{2}+\left(-s^{4}+4 s^{2}-1\right) t+1=0, s^{p} t^{q}=1\right\} .
\end{aligned}
$$

Let us denote the subsets of $\mathfrak{P}^{\prime \prime}$ corresponding to reducible and irreducible p-reps by $\left(\mathfrak{P}^{\prime \prime}\right)^{r} \subsetneq \mathfrak{F}^{r}$ and $\left(\mathfrak{P}^{\prime \prime}\right)^{i} \subsetneq \mathfrak{F}^{i}$, respectively, so that $\mathfrak{P}^{\prime \prime}=\left(\mathfrak{P}^{\prime \prime}\right)^{r} \cup\left(\mathfrak{P}^{\prime \prime}\right)^{i}$.

3.3. Eigenvalues of reducible p-reps. From equation (22), we can immediately read off the points corresponding to reducible representations as

$$
\mathfrak{F}^{r}=\left\{(s, 1, \pm 1,1) \mid s^{p}=1\right\},
$$

i.e. the eigenvalue $s$ is necessarily a $|p|$-th root of unity.

We now need to determine which of these points also correspond to p-reps $\rho \in$ $\mathfrak{R}\left(W_{p / q}\right)$, or equivalently to partial p-reps $\rho^{\prime} \in \mathfrak{R}(W)$.

The reader can easily verify (for example by using the inverse map defined in (15)) that a reducible representation $\rho^{\prime} \in \mathfrak{C}(W)$ corresponding to the eigenvalues $( \pm 1,1, \pm 1,1)$ is necessarily abelian and hence not a partial p-rep. On the other hand, all points $(s, 1, \pm 1,1) \in \mathfrak{F}^{r}$ with $s \neq \pm 1$ give indeed rise to partial p-reps: Since $u^{2}=t=v=1$ 
implies $\rho^{\prime}\left(\lambda_{0}\right)=I$, we have $\rho^{\prime}\left(\mu_{0}^{p} \lambda_{0}^{q}\right)=\rho^{\prime}\left(\mu_{0}^{p}\right)=I$ and hence $\rho^{\prime}$ factors through $\pi_{1}\left(W_{p / q}\right)$. Furthermore, $s^{2} \neq 1$ implies that $\rho^{\prime}$ is indeed non-abelian.

This means that the subset $\left(\mathfrak{P}^{\prime \prime}\right)^{r} \subsetneq \mathfrak{F}^{r}$ is given by

$$
\left(\mathfrak{P}^{\prime \prime}\right)^{r}=\left\{(s, 1, \pm 1,1) \mid s^{p}=1, s \neq \pm 1\right\} .
$$

To obtain the cardinality of this set, first note that -1 is a $|p|$-th root of unity iff $p$ is even. Since we always have two choices for $u= \pm 1$, we therefore obtain

$$
\#\left(\mathfrak{P}^{\prime \prime}\right)^{r}= \begin{cases}2(|p|-1) & \text { if } p \text { is odd } \\ 2(|p|-2) & \text { if } p \text { is even. }\end{cases}
$$

3.4. Eigenvalues of irreducible p-reps. Let us denote the defining polynomials of $\mathfrak{F}^{i}$ by

$$
\begin{aligned}
& k_{1}=s^{p} t^{q}-1, \\
& k_{2}=s^{4} t^{2}+\left(-s^{4}+4 s^{2}-1\right) t+1,
\end{aligned}
$$

so that $\mathfrak{F}^{i}=\left\{u^{2}=1, v=-1, k_{1}=k_{2}=0\right\}$.

To determine the solutions of $k_{1}=k_{2}=0$ we will use the resultant of $k_{1}$ and $k_{2}$ with respect to $t$, which we determine by induction on $q \geq 1$ as the determinant of the corresponding Sylvester matrix:

$$
\begin{aligned}
& \operatorname{res}_{p, q}(s):=\operatorname{Resultant}\left(k_{1}, k_{2}, t\right) \\
& =\operatorname{det}\left(\begin{array}{cccccc}
s^{p} & 0 & s^{4} & & & \\
0 & s^{p} & -s^{4}+4 s^{2}-1 & s^{4} & & \\
& 0 & 1 & -s^{4}+4 s^{2}-1 & & \\
& & 1 & \ddots & \\
\vdots & \vdots & & \ddots & s^{4} \\
-1 & 0 & & \ddots & -s^{4}+4 s^{2}-1 \\
0 & -1 & & & 1
\end{array}\right)_{(q+2) \times(q+2)} \\
& =s^{p+2 q}\left(s^{p-2 q}+(-1)^{q+1} 2 T_{q}(y)+s^{-p+2 q}\right) \\
& \doteq s^{p-2 q}+(-1)^{q+1} 2 T_{q}(y)+s^{-p+2 q} \text {, }
\end{aligned}
$$

where $y=y(s)=\frac{1}{2}\left(-s^{2}+4-s^{-2}\right)$, and $T_{q}(y)$ is the $q$-th Chebyshev polynomial of first type, satisfying $T_{0}(y)=1, T_{1}(y)=y$ and $T_{k+1}(y)=2 y T_{k}(y)-T_{k-1}(y)$ for $k \geq 1$ (see standard literature on orthogonal polynomials, e.g. [Sze75]).

Note that $\operatorname{res}_{p, q}$ is in fact a Laurent polynomial in $\mathbb{Z}\left[s^{ \pm 1}\right]$ when $p<0$, but we will often simply use the word polynomial to also include Laurent polynomials. Since we are only interested in solutions with $s \neq 0$, we will always regard this polynomial as being defined up to multiplication by a unit $s^{k}(k \in \mathbb{Z})$, which we indicate with the symbol $\doteq$. Since $T_{q}(y)$ is a polynomial of degree $q$ in $y$, the middle terms of $\operatorname{res}_{p, q}$ are formed by a symmetrical Laurent polynomial of span $4 q$ in the variable $s$, containing only even powers. Note that we can consider equation (28) as a formal definition for the case $p / q=1 / 0$, as then $\operatorname{res}_{1,0} \doteq(s-1)^{2}=0$ is equivalent to $k_{1}=0$, implying $s=1, t=-1$.

For us, the main importance of this resultant is that its roots characterize the solutions of the system $\left\{k_{1}=k_{2}=0\right\}$ : The extension theorem of elimination theory (cf. CLO97, $\S 1$, Theorem 3]) shows that the system $\left\{k_{1}=0, k_{2}=0\right\}$ has a solution $(s, t)$ if and only if $s \neq 0$ is a root of this resultant. We will call $s$ a partial solution in this case.

Furthermore note that given a partial solution $s$, the value for $t$ is uniquely determined by $k_{1}=0$ and $k_{2}=0$ : To see this, note that $k_{2}=0$ implies $t^{2}=-s^{-4}\left(\left(-s^{4}+4 s^{2}-1\right) t+1\right)$, 
and hence higher powers of $t$ can recursively be replaced by linear terms in $t$. The equation $k_{1}=s^{p} t^{q}-1=0$ can therefore always be expanded to an equation which is linear in $t$.

Denote the unique solution for $t$ for a given $s$ by $t_{s}$ - each root $s \neq 0$ of the resultant therefore corresponds to two eigenvalue points $\left(s, t_{s}, \pm 1,1\right)$ in $\mathfrak{F}^{i} \subset \mathfrak{E}_{0}(W)$, so that we have

$$
\mathfrak{F}^{i}=\left\{\left(s, t_{s}, \pm 1,1\right) \mid \operatorname{res}_{p, q}(s)=0\right\} .
$$

As in the reducible case, not all of these points correspond to representations which will factor through $\pi_{1}\left(W_{p / q}\right)$ to give p-reps in $\mathfrak{R}\left(W_{p / q}\right)$. This is due to the fact that the eigenvalue condition $s^{p} t^{q}=1$ does not necessarily imply that $\rho\left(\mu_{0}\right)^{p} \rho\left(\lambda_{0}\right)^{q}=I$. In fact, we have:

Proposition 8. The roots $s \notin\{0,1,-1\}$ of $\operatorname{res}_{p, q}$ correspond to irreducible p-reps of $\pi_{1}\left(W_{p / q}\right)$; the roots $s= \pm 1$ (when they arise) correspond to discrete faithful representations of $\pi_{1}(W)$ which therefore do not factor through $\pi_{1}\left(W_{p / q}\right)$.

Proof. For $s= \pm 1$, a direct calculation shows that an irreducible representation with $s^{2}=u^{2}=1$ is conjugate to the form

$$
\rho\left(\mu_{0}\right)=\left(\begin{array}{cc}
s & -s u \pm i \\
0 & s
\end{array}\right), \rho\left(\mu_{1}\right)=\left(\begin{array}{ll}
u & 0 \\
1 & u
\end{array}\right), \quad \text { and } \rho\left(\lambda_{0}\right)=\left(\begin{array}{cc}
-1 & -4 u \\
0 & -1
\end{array}\right),
$$

where $s, u \in\{ \pm 1\}$. (These are in fact, up to conjugation, all discrete faithful representations into $\mathrm{SL}_{2}(\mathbb{C})$ corresponding to the unique complete hyperbolic structure of the unfilled Whitehead link complement.) For each choice of $s= \pm 1$, there are four such representations. As the subgroup generated by the parabolic elements $\rho\left(\mu_{0}\right), \rho\left(\lambda_{0}\right) \in \mathrm{SL}_{2}(\mathbb{C})$ is therefore isomorphic to $\mathbb{Z}^{2}$, the equation $\rho\left(\mu_{0}\right)^{p} \rho\left(\lambda_{0}\right)^{q}=I$ can only be satisfied by $p=q=0$ (which is not a valid filling slope).

For $s \neq \pm 1$, first note that the inverse map (14) of the eigenvalue map is well defined (since $\left.u^{2}=1\right)$. The two points $\left(s, t_{s}, \pm 1,-1\right)$ associated to a root $s$ therefore correspond to two partial p-reps in $\mathfrak{C}(W)$. To see that these indeed factor through $\pi_{1}\left(W_{p / q}\right)$, note that for $s \neq \pm 1$, both $\rho\left(\mu_{0}\right)$ and $\rho\left(\lambda_{0}\right)$ are simultaneously diagonizable, since they are not parabolic and commute. The condition $s^{p} t^{q}=1$ then immediately implies $\rho\left(\mu_{0}\right)^{p} \rho\left(\lambda_{0}\right)^{q}=I$.

This concludes the proof of Theorem 3 For the following application to the minimal total Culler-Shalen seminorm in section 4, we now need to study the number of conjugacy classes of p-reps.

3.5. Roots of the resultant. We summarize some key facts about the roots of the resultant $\operatorname{res}_{p, q}$ :

Lemma 9 (Trivial roots). If $q$ is even (and hence $p$ odd), $s=1$ is a root of order 2 of $\operatorname{res}_{p, q}$. If both $q$ and $p$ are odd, $s=-1$ is a root of order 2 of $\operatorname{res}_{p, q}$. In the remaining case ( $q$ odd and $p$ even), neither of \pm 1 is a root of $\operatorname{res}_{p, q}$.

Proof. This can be directly verified from the equations $k_{1}=k_{2}=0$ (cf. (26) and (27)): If $s= \pm 1$, equation $k_{2}=0$ necessarily implies $t_{s}=-1$. Using $k_{1}=0$, we then immediately see that $(1,-1)$ is a solution iff $q$ is even, and that $(-1,-1)$ is a solution iff $p+q$ is even (which - under the assumption that $p$ and $q$ are coprime - is equivalent to both $p$ and $q$ being odd). To show that the order is equal to 2 , note that

$$
\operatorname{res}_{p, q}^{\prime}:=\frac{d}{d s} \operatorname{res}_{p, q}=(p-2 q)\left(s^{p-2 q-1}-s^{-p+2 q-1}\right)+(-1)^{q+1} 2 q U_{q-1}(y) y^{\prime}
$$

and

$$
\begin{gathered}
\operatorname{res}_{p, q}^{\prime \prime}=(p-2 q)\left((p-2 q-1) s^{p-2 q-2}+(-p+2 q-1) s^{-p+2 q-2}\right) \\
+(-1)^{q+1} 2 q\left(y^{\prime \prime} U_{q-1}(y)+\left(y^{\prime}\right)^{2} \frac{\mathrm{d}}{\mathrm{d} y} U_{q-1}(y)\right)
\end{gathered}
$$


where $U(y)$ is the Chebyshev polynomial of second type, satisfying $U_{0}(y)=1, U_{1}(y)=$ $2 y$ and $U_{k+1}(y)=2 y U_{k}(y)-U_{k-1}(y)$ for $k \geq 1$ (cf. Szze75]). Since $y^{\prime}( \pm 1)=0$, we immediately get $\operatorname{res}_{p, q}^{\prime}( \pm 1)=0$. Furthermore, $\operatorname{res}_{p, q}^{\prime \prime}( \pm 1) \neq 0$ unless $p / q=0 / 1$ or $p / q=$ $4 / 1$, but in those cases $\operatorname{res}_{p, q}$ degenerates and has no non-zero roots.

LEMma 10 (Symmetries).

(1) $\operatorname{res}_{p, q}(s)=0 \Longleftrightarrow \operatorname{res}_{p, q}\left(s^{-1}\right)=0$.

(2) $\operatorname{res}_{p, q}(s)=0 \Longleftrightarrow \operatorname{res}_{p, q}(\bar{s})=0$.

(3) $\left(\operatorname{res}_{p, q}(s)=0 \Longleftrightarrow \operatorname{res}_{p, q}(-s)=0\right)$ iff $p$ is even.

(4) $\operatorname{res}_{p, q}(s) \doteq \operatorname{res}_{-p+4 q, q}(s)$.

Proof. For (1), observe that $y\left(s^{-1}\right)=y(s)$. For $(2)$, note that $\operatorname{res}_{p, q}$ is a Laurent polynomial with real (in fact integer) coefficients. For $(3)$, first note that $y(-s)=y(s)$ and hence

$$
\operatorname{res}_{p, q}(-s)-\operatorname{res}_{p, q}(s)=\left((-1)^{p}-1\right)\left(s^{p-2 q}+s^{-p+2 q}\right) .
$$

This is clearly 0 when $p$ is even. Conversely, assume this difference to be 0 but $p$ to be odd. Then $s^{2(p-2 q)}=-1$, which implies $s=e^{i \phi}$ for some $0 \leq \phi<2 \pi$. But then $\operatorname{res}_{p, q}(s)=(-1)^{q+1} 2 T_{q}(y(s))=(-1)^{q+1} 2 T_{q}(2-\cos (2 \phi)) \neq 0$, since the roots of $T_{q}(y)$ lie in the interval $(0,1)$ (cf. [Sze75]). The last statement is obvious from (28).

Lemma 11 (Real roots). When $p>4 q>0$ or $p<0$, the only possible real-valued roots of $\operatorname{res}_{p, q}$ are \pm 1 . When $0<p<4 q$ and $p$ is odd, there are two additional real-valued, positive roots. When $0<p<4 q$ and $p$ is even (and hence $q$ odd), there are precisely four real-valued roots in total, none of which is \pm 1 .

Proof. Assume $\operatorname{res}_{p, q}(s)=0$ with real-valued $s>0$. Then

$$
s^{p-2 q}+s^{-(p-2 q)}=(-1)^{q} 2 T_{q}(y(s)) .
$$

Denote the left-hand side of this equation by $\phi(s)$, the right-hand side by $\psi(s)$. We will study intersections of these two real-valued functions. Note that $\phi(s)$ is strictly monotonously decreasing for $0<s<1$, has a global minimum of value 2 at $s=1$, and is strictly monotonously increasing for $s>1$. Furthermore, note that the range of $y(s)$ is $(-\infty, 1]$, with global maximum at $s=1$. This implies that the range of $\psi(s)=$ $(-1)^{q} 2 T_{q}(y(s))$ is $(-\infty, 2)$, using the fact that $T_{q}(y)$ is a polynomial of degree $q$ in $y$, whose zeros and relative extrema of value \pm 1 are all contained in the interval $[-1,1]$. More specifically, $\psi$ oscillates with values between -2 and 2 for $s \in[1-\sqrt{2}, 1+\sqrt{2}]$ (with $\psi(1)=(-1)^{q} 2$ ) and tends strictly monotonously to $+\infty$ for $s \rightarrow 0$ and $s \rightarrow \infty$.

Apart from the possibility of an intersection at $s=1$ (in the case that $q$ is even), other intersections between $\phi$ and $\psi$ can therefore only occur outside the interval $(1-\sqrt{2}, 1+\sqrt{2})$ when the maximal degree in $s$ of $\psi$ is larger than that of $\phi$. But $\operatorname{maxdeg}_{s} \phi=|p-2 q|=$ $-\operatorname{mindeg}_{s} \phi$ and $\operatorname{maxdeg}_{s} \psi=2 q=-\operatorname{mindeg}_{s} \psi$. It is then easy to see that for $p>4 q>0$ or $p<0, \operatorname{maxdeg}_{s} \psi<\operatorname{maxdeg}_{s} \phi$ and there are no such intersections. The same argument then shows that for $0<p<4 q$, there are precisely two positive real-valued roots different from 1 (which are inverses of each other, by Lemma 10 (10).

To analyze the remaining case $s<0$, note that $\phi(-s)=(-1)^{p} \phi(s)$ while $\psi(-s)=$ $\psi(s)$. If $p$ is odd, this means that there cannot be any intersection apart from possibly $s=-1$ (which arises if $q$ is odd), as the two functions diverge in opposite directions. If $p$ is even (and hence $q$ odd), we get as roots the negatives of the roots found for $s>0$. In total, there are either 0 or 4 real roots in this case, as $\operatorname{res}_{p, q}( \pm 1) \neq 0$.

Note that this includes the case $p / q=2 / 1$, when there are only four non-zero roots in total, all of which are real and distinct from \pm 1 . 
Lemma 12 (Imaginary roots). When $p>4 q>0$ or $p<0$, $\operatorname{res}_{p, q}$ has imaginary roots iff $p \in 4 \mathbb{Z}$. In that case, there are four such roots. When $0<p<4 q$, there are no imaginary roots.

Proof. Similar to the proof of Lemma 11 Assume $\operatorname{res}_{p, q}(i x)=0$ with $x>0$. Then

$$
i^{-p+2 q}\left((-1)^{p} x^{p-2 q}+x^{-p+2 q}\right)=(-1)^{q} 2 T_{q}\left(\frac{x^{2}+4+x^{-2}}{2}\right) .
$$

Now note that the right-hand side is always real-valued, while the same for the left-hand side is only true when $p$ is even. In that case, the equation is equivalent to

$$
\phi(x):=(-1)^{p / 2}\left(x^{p-2 q}+x^{-p+2 q}\right)=2 T_{q}\left(\frac{x^{2}+4+x^{-2}}{2}\right)=: \psi(x) .
$$

As $\psi(x)>0$ for all $x \in \mathbb{R}$, this equation can only be satisfied if $p / 2$ is even, i.e. if $p \in 4 \mathbb{Z}$. Under this assumption, comparison of the maximal degrees then shows that there is no intersection of $\phi$ and $\psi$ when $0<p<4 q$, and precisely two intersections (which are inverses of each other) when $p>4 q>0$ or $p<0$. The symmetry with respect to complex conjugation then shows that there are in total four pure imaginary roots in this case, and none when $p \notin 4 \mathbb{Z}$.

Lemma 13. None of the roots $s \notin\{ \pm 1\}$ of $\operatorname{res}_{p, q}$ lies on the unit circle.

Proof. Simply note that for $\phi \in[0,2 \pi)$,

$$
\operatorname{res}_{p, q}\left(e^{i \phi}\right)=2(\underbrace{\cos (p-2 q) \phi}_{\in[-1,1]}+(-1)^{q+1} \underbrace{T_{q}(2-\cos 2 \phi)}_{\geq 1})=0
$$

implies $\phi=0$ or $\phi=\pi$.

Examples of the typical distribution of the roots are shown in figures 2(a) 2(b)] and 2(c) on page 15

We will now determine the number of non-zero roots of the resultant. The number of non-zero roots (with multiplicities) of a Laurent polynomial is given by its span, i.e. the difference of the maximal and minimal degrees. From (28), we see that unless $p / q \in\{0,4\}$, the span of $\operatorname{res}_{p, q}$ is $2 \max (|p-2 q|, 2 q)$ (recall that we always assume $q>0$ ). When $p / q \in\{0,4\}, \operatorname{res}_{p, q} \doteq 1$, and there are therefore no irreducible p-preps - matching the fact that $W_{0}$ and $W_{4}$ are toroidal manifolds which do not admit a complete hyperbolic structure.

Discarding 0, 1 and -1 from the roots of $\operatorname{res}_{p, q}$, we therefore obtain an upper bound for the number of distinct non-trivial roots as given in table 4 . We remark that it is a hard problem to analytically show that all non-trivial roots are in fact simple - but the proof of Theorem 1 will in fact imply this for odd $p$ (cf. Corollary 4).

\begin{tabular}{c||ccc}
$p / q \in$ & $(-\infty, 0)$ & $(0,4)$ & $(4, \infty)$ \\
\hline$p$ odd & $2|p|+4|q|-2$ & $4|q|-2$ & $2|p|-4|q|-2$ \\
$p$ even & $2|p|+4|q|$ & $4|q|$ & $2|p|-4|q|$
\end{tabular}

TABLE 4. Upper bound for the number of distinct roots $s \notin$ $\{0, \pm 1\}$ of $\operatorname{res}_{p, q}$ 




(a) $\operatorname{res}_{65,3}(p>4 q>0)$

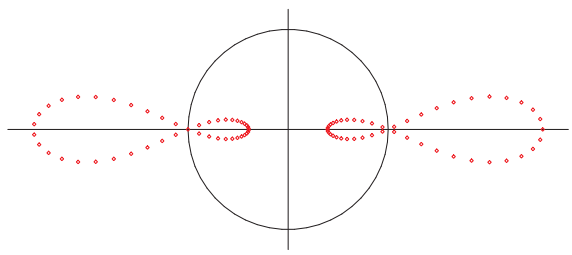

(b) $\operatorname{res}_{65,23}(0<p<4 q)$

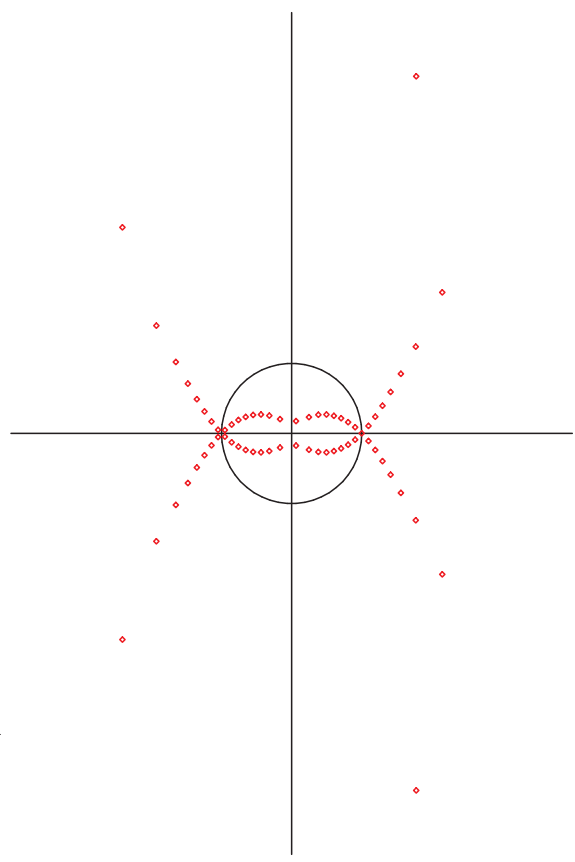

(c) $\operatorname{res}_{65,16}(p-4 q>0$, but small)

Figure 2. Examples of the distribution of the non-zero roots of $\operatorname{res}_{p, q}$

3.6. The number of conjugacy classes of p-reps. We will now put together the results of the previous sections to obtain the count of conjugacy classes of p-reps for the manifold $W_{p / q}$.

We have determined the two components of the set $\mathfrak{P}^{\prime \prime}$ of eigenvalue tuples corresponding to p-reps as

$$
\begin{aligned}
\left(\mathfrak{P}^{\prime \prime}\right)^{r} & =\left\{(s, \pm 1,1,1) \mid s^{p}=1, s \neq \pm 1\right\}, \\
\left(\mathfrak{P}^{\prime \prime}\right)^{i} & =\left\{\left(s, \pm 1, t_{s},-1\right) \mid \operatorname{res}_{p, q}(s)=0, s \neq \pm 1\right\},
\end{aligned}
$$

with their cardinalities given in equation (25) and table 4, respectively.

Now recall that by construction, the cardinality of $\mathfrak{P}^{\prime \prime}$ is equal to the cardinality of $\mathfrak{P}^{\prime}$ and that of $\mathfrak{P}$. Furthermore, we saw that each conjugacy class of p-reps in $\mathfrak{C}\left(W_{p / q}\right)$ is represented by two elements in $\mathfrak{C}\left(W_{p / q}\right)$ (corresponding to the pair of eigenvalue points $(s, t, u, v)$ and $\left.\left(s^{-1}, t^{-1}, u, v\right)\right)$. To obtain the number of conjugacy classes of p-reps, we therefore have to divide the cardinality of $\mathfrak{P}$ by 2 . Since the two representatives for a conjugacy class in $\mathfrak{C}\left(W_{p / q}\right)$ clearly have the same character independent of whether they are reducible or irreducible, we see that this number also coincides with the number of characters of p-reps.

This then shows:

Proposition 14. The number of distinct conjugacy classes of $\mathrm{SL}_{2}(\mathbb{C})$ p-reps of $\pi_{1}\left(W_{p / q}\right)$ is bounded above by the numbers shown in table 5 : 


\begin{tabular}{c||ccc}
$p / q \in$ & $(-\infty, 0)$ & $(0,4)$ & $(4, \infty)$ \\
\hline$p$ odd & $3|p|+4|q|-3$ & $|p|+4|q|-3$ & $3|p|-4|q|-3$ \\
$p$ even & $3|p|+4|q|-2$ & $|p|+4|q|-2$ & $3|p|-4|q|-2$
\end{tabular}

TABLE 5. Upper bound for the number of conjugacy classes of $\mathrm{SL}_{2}(\mathbb{C})$ p-reps of $\pi_{1}\left(W_{p / q}\right)$

\section{The minimal total Culler-Shalen seminorm and p-reps}

The goal of this section is to show:

ThEOREM 15. The minimal total Culler-Shalen seminorm of the manifold $W_{p / q}$ is equal to the number of characters of $p$-reps of $\pi_{1}\left(W_{p / q}\right)$. In particular, it is less than or equal to the bounds given in Table 5 .

Since Dehn filling along the meridian $\mu_{1}$ of $W_{p / q}$ gives a closed manifold with cyclic fundamental group, while $\mu_{1}\left(=\frac{1}{0}=\infty\right)$ is not a boundary slope, Corollary 1.1 .4 of CGLS87 implies that its Culler-Shalen seminorm is in fact equal to the minimal total Culler-Shalen seminorm $s$ :

$$
s=\left\|\mu_{1}\right\|=\|\infty\| .
$$

We can therefore find $s$ by explicitly determining $\left\|\mu_{1}\right\|$.

Recall that $\left\|\mu_{1}\right\|=\operatorname{deg}\left(f_{\mu_{1}}: \widetilde{\mathfrak{X}}^{\operatorname{irr}}\left(W_{p / q}\right) \rightarrow \mathbb{C P}^{1}\right)$. We will determine this degree as a count of the roots of $f_{\mu_{1}}$. First note that we can in fact restrict our attention to $\mathfrak{X}\left(W_{p / q}\right)$, since $f_{\mu_{1}}$ has poles at each ideal point in $\widetilde{\mathfrak{X}}\left(W_{p / q}\right)$ (see e.g. the remarks after Corollary 9.2.7 in Sha02 $)$.

Since $f_{\mu_{1}}=0$ is equivalent to $\chi_{\rho}\left(\mu_{1}\right)=$ trace $\rho\left(\mu_{1}\right)= \pm 2$, we immediately see that zeroes of this function are characters of representations $\rho \in \mathfrak{R}\left(W_{p / q}\right)$ which send the meridian $\mu_{1} \in \pi_{1}\left(W_{p / q}\right)$ to $\pm I$ or a parabolic element of $\mathrm{SL}_{2}(\mathbb{C})$. Since we only regard non-trivial curves in $\mathfrak{X}\left(W_{p / q}\right)$, the representations in question have to be non-abelian, and are therefore $p$-reps.

Theorem [15] will therefore be a direct consequence of the following two propositions, which are the subject of the bulk of this section:

Proposition 16. Each character of a $p$-rep of $\pi_{1}\left(W_{p / q}\right)$ is a smooth point of a unique non-trivial curve in $\mathfrak{X}^{\text {irr }}\left(W_{p / q}\right)$.

Proposition 17. Each character of an irreducible p-rep of $\pi_{1}\left(W_{p / q}\right)$ is a simple zero of $f_{\mu_{1}}$. If $p / q \neq 0$, also each character of a non-abelian reducible $p$-rep of $\pi_{q}\left(W_{p / q}\right)$ is a simple zero of $f_{\mu_{1}}$.

We will begin in section 4.1 by using work of Heusener and Porti (cf. HP05) to show Proposition 16 for a general non-abelian reducible representation (therefore including the case of reducible p-reps). We remark that this is a subtle question, as it asks when the character of a non-abelian reducible representation can be deformed into the character of an irreducible representation. This result will also be needed in section 5

To show Proposition [16 for an irreducible p-rep, we then exhibit a useful partiallydiagonal normal form for p-reps in section 4.2 and use this in section 4.3 to determine an explicit presentation of the cohomology of $\pi_{1}\left(W_{p / q}\right)$ with coefficients in $\mathfrak{s l}_{2}^{\rho}$ (the Lie algebra $\mathfrak{s l}_{2}(\mathbb{C})$ viewed as a $\pi_{1}\left(W_{p / q}\right)$ module via the map Ad $\circ \rho$ for a given p-rep $\rho$ ). The knowledge of the dimension of this cohomology group then implies our claim using Theorem A of Boy02.

Finally, Proposition 17 will be shown in section 4.4 by studying the degree of the trace function $f_{\mu_{1}}$ in relation to deformations of representations. 
4.1. Smoothness of the characters of general non-abelian reducible representations. We will show:

Proposition 18. Let $\bar{\rho} \in \overline{\mathfrak{R}}\left(W_{p / q}\right)$ be a non-abelian reducible $\mathrm{PSL}_{2}(\mathbb{C})$ representation. For $p / q \neq 4$, the character $\chi_{\bar{\rho}}$ of $\bar{\rho}$ is contained in precisely two irreducible components of $\overline{\mathfrak{X}}\left(W_{p / q}\right)$, both of which are curves, one trivial, the other non-trivial. In addition, $\chi_{\bar{\rho}}$ is a smooth point of both curves and the intersection at $\chi_{\bar{\rho}}$ is transverse. When $p / q=4$, the above is true for all non-abelian reducible representations $\bar{\rho}$ with trace $\bar{\rho}\left(\mu_{0}\right) \neq \pm 2$.

Since every non-abelian reducible $\mathrm{SL}_{2}(\mathbb{C})$ representation $\rho \in \mathfrak{R}\left(W_{p / q}\right)$ induces a nonabelian reducible $\mathrm{PSL}_{2}(\mathbb{C})$ representation $\bar{\rho} \in \overline{\mathfrak{R}}\left(W_{p / q}\right)$, this then implies the following stronger version of Proposition 16 in the case of non-abelian reducible representations:

COROLlaRY 19. Every non-abelian reducible $\mathrm{SL}_{2}(\mathbb{C})$ character $\chi_{\rho} \in \mathfrak{X}\left(W_{p / q}\right)$ is a smooth point on a unique non-trivial curve $\mathfrak{X}_{0} \subset \mathfrak{X}\left(W_{p / q}\right)$.

Proof. Let $\rho \in \mathfrak{R}\left(W_{p / q}\right)$ be a non-abelian reducible representation, and let $\bar{\rho} \in$ $\bar{\Re}\left(W_{p / q}\right)$ be its image under the natural projection $\pi: \mathfrak{R}\left(W_{p / q}\right) \rightarrow \bar{\Re}\left(W_{p / q}\right)$. Proposition 18 now shows that $\bar{\rho}$ lies as a smooth point on a unique non-trivial curve $\overline{\mathfrak{X}}_{0} \subset$ $\overline{\mathfrak{X}}\left(W_{p / q}\right)$, and we only need to show that this curve lifts to a non-trivial curve $\mathfrak{X}_{0} \subset$ $\mathfrak{X}\left(W_{p / q}\right)$. But this is immediately clear, since $\bar{\rho}$ lifts to $\rho$ by construction, and $\pi$ is a regular covering map onto its image (cf. [BZ98, Section 3]).

We start by determining all possible non-abelian reducible representations of $\pi_{1}\left(W_{p / q}\right)$ up to conjugation:

Lemma 20. Let $\bar{\rho}$ be a non-abelian reducible representation in $\bar{\Re}\left(W_{p / q}\right)$. Then up to conjugation, $\bar{\rho}$ has the form

$$
\bar{\rho}\left(\mu_{0}\right)= \pm\left(\begin{array}{cc}
s & 0 \\
c & s^{-1}
\end{array}\right) \quad \text { and } \quad \bar{\rho}\left(\mu_{1}\right)= \pm\left(\begin{array}{cc}
u & 0 \\
d & u^{-1}
\end{array}\right),
$$

where either

(1) $s^{2}=1, c \neq 0$ and $u^{2}=\frac{1}{2 q}(-p+2 q \pm \sqrt{p(p-4 q)})$ (where $p / q=0$ cannot arise), or

(2) $u^{2}=1, d \neq 0$ and $s^{p}= \pm 1$, but $s^{2} \neq 1$.

Proof. Let $\bar{\rho} \in \bar{\Re}\left(W_{p / q}\right)$ be non-abelian reducible. Since it is reducible, we can conjugate it into lower triangular form as in the claim. The first group relation of $\pi_{1}\left(W_{p / q}\right)$ (cf. equation (6) ) now implies that either $d u\left(1-s^{2}\right)+c s\left(u^{2}-1\right)=0$ (in which case $\bar{\rho}$ would be abelian, a contradiction), or one of $s^{2}=1$ or $u^{2}=1-$ but not both (otherwise $\bar{\rho}$ would again be abelian).

If $s^{2}=1$, we hence have $u^{2} \neq 1$ and $c \neq 0$. We can now conjugate by diagonal

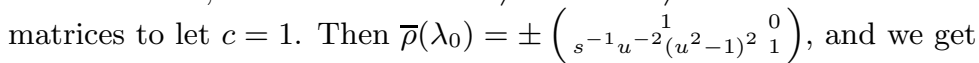

$$
\begin{aligned}
\pm I=\bar{\rho}\left(\mu_{0}\right)^{p} \bar{\rho}\left(\lambda_{0}\right)^{q} & = \pm\left(\begin{array}{cc}
s^{p} & 0 \\
s^{p-1} p & s^{p}
\end{array}\right)\left(\begin{array}{cc}
1 & 0 \\
q s^{-1} u^{-2}\left(u^{2}-1\right)^{2} & 1
\end{array}\right) \\
& = \pm\left(\begin{array}{cc}
s^{p} & 0 \\
p s^{p-1}+s^{p} q s^{-1} u^{-2}\left(u^{2}-1\right)^{2} & s^{p}
\end{array}\right) .
\end{aligned}
$$

Since $s^{2}=1$, the lower left entry implies $q u^{4}+u^{2}(p-2 q)+q=0$, and therefore $u^{2}=$ $\frac{1}{2 q}(-p+2 q \pm \sqrt{p(p-4 q)})$ as claimed. Note that these roots are distinct unless $p / q=0$ or $p / q=4$. When $p / q=0$, this implies that $u^{2}=1$, a contradiction since $\bar{\rho}$ is non-abelian. When $p / q=4$, we get $u^{2}=-1$, which yields a valid non-abelian reducible representation.

In the remaining case, when $s^{2} \neq 1$, we have $u^{2}=1$ and can assume $d=1$. Then $\bar{\rho}\left(\lambda_{0}\right)= \pm I$ and we immediately get $\pm I=\bar{\rho}\left(\mu_{0}\right)^{p} \bar{\rho}\left(\lambda_{0}\right)^{q}=\bar{\rho}\left(\mu_{0}\right)^{p}$ which implies $s^{p}=$ \pm 1 . 
Following [HP05], we now determine the twisted Alexander polynomial associated to the diagonal representation $\bar{\rho}_{\alpha}$ obtained from a non-abelian reducible representation $\bar{\rho} \in \overline{\mathfrak{R}}\left(W_{p / q}\right)$ as in Lemma 20 by setting all non-diagonal matrix entries to be zero. In particular, this representation has the same character as $\bar{\rho}$, i.e. $\chi_{\bar{\rho}_{\alpha}}=\chi_{\bar{\rho}}$.

LEMma 21. Let $\bar{\rho}$ be a non-abelian reducible representation in $\bar{\Re}\left(W_{p / q}\right)$, and let $\bar{\rho}_{\alpha}$ be the diagonal representation with the same character as $\bar{\rho}$ as described above. The twisted Alexander polynomial associated to $\rho_{\alpha}$ is given by:

$$
\Delta^{\Phi_{\sigma}}(t) \doteq \begin{cases}t-1 & \text { if } s^{2} \neq 1 \\ q t^{2}+(p-2 q) t+q & \text { if } s^{2}=1\end{cases}
$$

Proof. We follow the notation of HP05 to facilitate comparison for the reader. First note that $H_{1}\left(W_{p / q} ; \mathbb{Z}\right) \cong \mathbb{Z} / p \oplus \mathbb{Z}=\left\langle\mu_{0}\right\rangle \oplus\left\langle\mu_{1}\right\rangle$, where we use the same symbols for the generators of $\pi_{1}\left(W_{p / q}\right)$ and their images under abelianization. We now define the following homomorphisms:

$$
\begin{array}{lll}
\alpha: \pi_{1}\left(W_{p / q}\right) \rightarrow H_{1}\left(W_{p / q} ; \mathbb{Z}\right) \rightarrow \mathbb{C}^{*}, & \mu_{0} \mapsto s^{2}, & \mu_{1} \mapsto u^{2}, \\
\phi: \pi_{1}\left(W_{p / q}\right) \rightarrow \mathbb{Z}=\langle t\rangle, & \mu_{0} \mapsto 1, & \mu_{1} \mapsto t, \\
\sigma: \pi_{1}\left(W_{p / q}\right) \rightarrow \text { tors } H_{1}\left(W_{p / q} ; \mathbb{Z}\right) \stackrel{\alpha}{\rightarrow} \mathbb{C}^{*}, & \mu_{0} \mapsto s^{2}, & \mu_{1} \mapsto 1,
\end{array}
$$

where $s$ and $u$ are the upper left entries of $\bar{\rho}\left(\mu_{0}\right)$ and $\bar{\rho}\left(\mu_{1}\right)$, respectively. Using these maps, we can now define a map $\Phi_{\sigma}: \pi_{1}\left(W_{p / q}\right) \rightarrow \mathbb{C}\left[t^{ \pm}\right]^{*}$ to the units of the ring of Laurent polynomials $\mathbb{C}\left[t^{ \pm 1}\right]$ by $\Phi_{\sigma}(\gamma):=\sigma(\gamma) t^{\phi(\gamma)}$ for $\gamma \in \pi_{1}\left(W_{p / q}\right)$. This map can be linearly extended to the group ring $\mathbb{Z} \pi_{1}\left(W_{p / q}\right)$ which allows us to define the twisted Alexander polynomial $\Delta^{\Phi_{\sigma}}(t) \in \mathbb{C}\left[t^{ \pm 1}\right]$ (cf. [HP05, Section 2] or e.g. Wad94 for more background). Since we have a presentation with two generators $\mu_{0}$ and $\mu_{1}$ and two relators

$$
r_{1}=\mu_{0} \mu_{1} \mu_{0}^{-1} \mu_{1}^{-1} \mu_{0}^{-1} \mu_{1} \mu_{0} \mu_{1} \mu_{0}^{-1} \mu_{1}^{-1} \mu_{0} \mu_{1} \mu_{0} \mu_{1}^{-1} \mu_{0}^{-1} \mu_{1}^{-1}
$$

and $r_{2}=\mu_{0}^{p} \lambda_{0}^{q}$, the twisted Alexander polynomial can be obtained as the greatest common divisor of the entries of the twisted Fox Jacobian $J^{\Phi_{\sigma}}$ :

$$
\begin{aligned}
J^{\Phi_{\sigma}} & =\left(\begin{array}{ll}
\frac{\partial r_{1}}{\partial \mu_{0}} & \frac{\partial r_{1}}{\partial \mu_{1}} \\
\frac{\partial r_{2}}{\partial \mu_{0}} & \frac{\partial r_{2}}{\partial \mu_{1}}
\end{array}\right)^{\Phi_{\sigma}} \\
& =\left(\begin{array}{cc}
s^{-2} t^{-1}(t-1)^{2}\left(s^{2}-1\right) & s^{-2} t^{-1}(1-t)\left(s^{2}-1\right)^{2} \\
\sum_{k=0}^{p-1} s^{2 k}+s^{2 p} q t^{-1}(t-1)^{2} & s^{2 p} q t^{-1}(1-t)\left(s^{2}-1\right)
\end{array}\right) .
\end{aligned}
$$

This matrix can be obtained by hand by first determining the Fox derivatives of the group relators (cf. Fox53, Ch. 9B] or BZ03 for background on Fox free differential calculus), and then applying the map $\Phi_{\sigma}$ to the obtained elements of the group ring $\mathbb{Z} \pi_{1}\left(W_{p / q}\right)$.

We now have two cases: If $s^{2}=1$, we get

$$
J^{\Phi_{\sigma}}=\left(\begin{array}{cc}
0 & 0 \\
p+q t^{-1}(t-1)^{2} & 0
\end{array}\right),
$$

and thus that $\Delta^{\Phi_{\sigma}}(t)=p+q t^{-1}(t-1)^{2} \doteq q t^{2}+(p-2 q) t+q$ (where $\doteq$ denotes equivalence up to multiplication by units of $\left.\mathbb{C}\left[t^{ \pm 1}\right]\right)$. Note that this is in fact the untwisted Alexander polynomial of $W_{p / q}$, as $s^{2}=1$ implies that $\sigma$ is the trivial 1-dimensional representation (cf. also e.g. HMW92, taking into account that the authors work with the left-handed Whitehead link).

If on the other hand $s^{2} \neq 1$, we have $s^{2 p}=1$ and $\sum_{k=0}^{p-1} s^{2 k}=0$ which yields

$$
J^{\Phi_{\sigma}}=\left(\begin{array}{cc}
s^{-2} t^{-1}(t-1)^{2}\left(s^{2}-1\right) & s^{-2} t^{-1}(1-t)\left(s^{2}-1\right)^{2} \\
q t^{-1}(t-1)^{2} & q t^{-1}(1-t)\left(s^{2}-1\right)
\end{array}\right) .
$$

As a result, $\Delta^{\Phi_{\sigma}}(t)=t^{-1}(t-1) \doteq t-1$ in this case. 
We can now proceed to prove the main result:

Proof of Proposition 18, Let $\bar{\rho} \in \bar{\Re}\left(W_{p / q}\right)$ be non-abelian reducible. Lemma 20 shows that $s^{2} \neq 1$ implies $u^{2}=1$ (i.e. $u^{2}$ is the root of the polynomial $t-1$ ), and $s^{2}=1$ implies $u^{2}$ is a root of $q t^{2}+(p-2 q) t+q$. But these are precisely the twisted Alexander polynomials found in Lemma 21 for these cases, and so we have that $u^{2}$ is a simple root of the twisted Alexander polynomial, unless $s^{2}=1$ and $p / q=4$ (in which case $u^{2}=-1$ is a double root). Apart from this exception, Theorems 1.2 and 1.3 of HP05 (with $\delta=\mu_{1}$ ) then show that the character of the diagonal representation $\bar{\rho}_{\alpha}$ (which is equal to the character of $\bar{\rho}$ ) satisfies the claim of the Proposition.

4.2. A partially diagonal parametrization of p-reps. We determine a parametrization of p-reps similar to the normal form (9) from section 3.1 but which is better suited to the following cohomology calculations.

Let $\rho \in \mathfrak{R}\left(W_{p / q}\right) \subset \mathfrak{R}(W)$ be a p-rep. We therefore can assume that $\rho\left(\mu_{0}\right)$ is not parabolic, and that furthermore trace $\rho\left(\mu_{1}\right)= \pm 2$.

For clarity of exposition, we will in the following restrict ourselves to the case trace $\rho\left(\mu_{1}\right)=$ +2 , and we attach the symbol "+" to all related sets and expressions to indicate this choice of sign. The negative trace case proceeds completely analogously and will be omitted here.

As a consequence, $\rho$ can be conjugated into the form

$$
\rho\left(\mu_{0}\right)=\left(\begin{array}{cc}
s & 0 \\
0 & s^{-1}
\end{array}\right) \quad \text { and } \quad \rho\left(\mu_{1}\right)=\left(\begin{array}{cc}
a & -(a-1)^{2} \\
1 & 2-a
\end{array}\right)
$$

where $s \in \mathbb{C}^{*}, a \in \mathbb{C}$, subject to the condition (obtained from applying (34) to the first group relation)

$$
r_{1}^{+}=(a-1)\left[-\left(s^{2}-1\right)^{2} a^{2}+\left(s^{2}-1\right)\left(s^{2}-3\right) a-2\right]=0 .
$$

Note that this implies $a \neq 0$ and $a \neq 2$ (otherwise $r_{1}^{+}=2 s^{4} \neq 0$ ).

We will in the following use this condition to simplify all arising polynomials modulo $r_{1}^{+}$, i.e. we will replace each polynomial $f$ by its canonical form $f_{0}$ modulo the ideal generated by $r_{1}^{+}$, in notation $f \equiv{ }_{r_{1}^{+}} f_{0}$ (see e.g. [CLO97]). We also extend this to rational functions by setting $f / g \equiv{ }_{r_{1}^{+}} f_{0} / g$ if $f \equiv_{r_{1}^{+}} f_{0}$.

Using this parametrization, we then obtain for the longitude that $\rho\left(\lambda_{0}\right)=\left(\begin{array}{cc}t & 0 \\ 0 & t^{-1}\end{array}\right)$ with $t \equiv_{r_{1}} a /(2-a)$, which implies that $\rho$ is diagonal on the whole peripheral subgroup of the first boundary torus. This fact will be of great importance in section 4.3

Applying (34) to the filling relation, $\rho\left(\mu_{0}^{p} \lambda_{0}^{q}\right)=I$, now yields

$$
r_{2}^{+} \equiv_{r_{1}^{+}} s^{p}\left(\frac{a}{2-a}\right)^{q}-1=0 .
$$

Note that $t \neq 0$ implies $a \neq 0$ and $a \neq 2$.

Denote by $\mathfrak{D}^{+}\left(W_{p / q}\right) \subset \mathfrak{R}\left(W_{p / q}\right)$ the subset of representations in partially-diagonal normal form (34) satisfying $r_{1}^{+}=r_{2}^{+}=0$.

Now note that $\rho \in \mathfrak{D}^{+}\left(W_{p / q}\right)$ is reducible iff $a=1$. In this case the polynomial $r_{1}^{+}$is identically zero, and poses therefore no further condition on $s$ and $a$. Then $\rho \in$ $\mathfrak{C}\left(W_{p / q}\right) \cap \mathfrak{D}^{+}\left(W_{p / q}\right)$, and $a=u=1$ is equal to the eigenvalue of $\rho\left(\mu_{1}\right)$. The condition $r_{2}^{+}=0$ then implies $s^{p}=1$, just as we found in section 3

If $\rho \in \mathfrak{D}^{+}\left(W_{p / q}\right)$ is irreducible, we have $a \neq 1$, and we can further simplify $r_{1}^{+}$to:

$$
r_{1}^{+}=-\left(s^{2}-1\right)^{2} a^{2}+\left(s^{2}-1\right)\left(s^{2}-3\right) a-2 .
$$

For simplicity, we will denote this polynomial again by $r_{1}^{+}$, since there will be no danger of confusion. Now note that the system $r_{1}^{+}=r_{2}^{+}=0$ (for the coefficients $s$ and $a$ ) is completely equivalent to the system $k_{1}=k_{2}=0$ (for eigenvalues $s$ and $t$ ) for p-reps (with positive trace on $\rho\left(\mu_{1}\right)$, i.e. $u=+1$ ) in section 3 , where we showed that its solutions are characterized by the roots of the polynomial $\operatorname{res}_{p, q}$. Instead of explicitly solving this 
system in the new parametrization, we will therefore use the properties of $\operatorname{res}_{p, q}$ where appropriate (in particular in section 4.4).

4.3. The twisted cohomology and smoothness at p-reps. This section is dedicated to proving Proposition 16 for irreducible p-reps by determining an explicit presentation for the cohomology group $H^{1}:=H^{1}\left(W_{p / q} ; \mathfrak{s l}_{2}^{\rho}\right)=H^{1}\left(\pi_{1}\left(W_{p / q}\right) ; \mathfrak{s l}_{2}^{\rho}\right)$. Good background references are [Bro82] and [Por97], we also recommend the articles [HPSP01] and HP05] for a good summary in our context.

4.3.1. Preliminaries. Consider the Lie group $\mathrm{SL}_{2}(\mathbb{C})$ and its Lie algebra $\mathfrak{s l}_{2}(\mathbb{C})$, and denote the adjoint homomorphism by $\mathrm{Ad}: \mathrm{SL}_{2}(\mathbb{C}) \rightarrow \operatorname{Aut}\left(\mathfrak{s l}_{2}(\mathbb{C})\right)$ acting by conjugation, i.e. for $A \in \mathrm{SL}_{2}(\mathbb{C})$ and $U \in \mathfrak{s l}_{2}(\mathbb{C})$ we have $\operatorname{Ad}(A)(U)=A U A^{-1}$.

Let $\Gamma$ be a finitely presented group, and let $\rho \in \mathfrak{R}(\Gamma)=\operatorname{Hom}\left(\Gamma ; \mathrm{SL}_{2}(\mathbb{C})\right)$. The Lie algebra $\mathfrak{s l}_{2}(\mathbb{C})$ can then be viewed as a (left) $\Gamma$ module via the map Ad $\circ \rho$, i.e. for $\gamma \in \Gamma$ and $A \in \mathrm{SL}_{2}(\mathbb{C})$ we have the left action $\gamma \cdot A:=\operatorname{Ad}(\rho(\gamma))(A)$. Denote $\mathfrak{s l}_{2}(\mathbb{C})$ with this left $\Gamma$ module structure by $\mathfrak{s l}_{2}^{\rho}$.

Denote by $C^{*}\left(\Gamma ; \mathfrak{s l}_{2}^{\rho}\right)$ the space of cochains with coefficients in $\mathfrak{s i}_{2}^{\rho}$. Similarly, let $B^{*}\left(\Gamma ; \mathfrak{s l}_{2}^{\rho}\right)$ and $Z^{*}\left(\Gamma ; \mathfrak{s}_{2}^{\rho}\right)$ denote the coboundaries and cocycles, respectively, and $H^{*}\left(\Gamma ; \mathfrak{s i}_{2}^{\rho}\right)$ the cohomology group with coefficients in $\mathfrak{s i}_{2}^{\rho}$.

Note that a cocycle $d \in Z^{1}\left(\Gamma ; \mathfrak{s l}_{2}^{\rho}\right)$ is a map $d: \Gamma \rightarrow \mathfrak{s l}_{2}^{\rho}$ satisfying

$$
d\left(\gamma_{1} \gamma_{2}\right)=d\left(\gamma_{1}\right)+\gamma_{1} \cdot d\left(\gamma_{2}\right) \quad \text { for all } \gamma_{1}, \gamma_{2} \in \Gamma,
$$

using the short notation for the Ad $\circ \rho$ action of $\Gamma$ on $\mathfrak{s l}_{2}(\mathbb{C})$. A cochain $b: \Gamma \rightarrow \mathfrak{s l}_{2}^{\rho}$ is a coboundary if there exists an $U \in \mathfrak{s l}_{2}(\mathbb{C})$ such that $b(\gamma)=\gamma \cdot U-U$ for all $\gamma \in \Gamma$.

It was an observation of Weil (Wei64], cf. also Por97]) that the Zariski tangent space $T_{\rho}^{\mathrm{Zar}}(\mathfrak{R}(\Gamma))$ at a point $\rho \in \mathfrak{R}(\Gamma)$ can be identified with a subspace of the space of 1-cocycles $Z^{1}\left(G ; \mathfrak{s l} \mathfrak{l}_{2}^{\rho}\right)$, and thus $\operatorname{dim} T_{\rho}^{\operatorname{Zar}}(\mathfrak{R}(\Gamma)) \leq \operatorname{dim} Z^{1}\left(\Gamma ; \mathfrak{s l}_{2}^{\rho}\right)$. The knowledge of the dimension of the space of cocycles can therefore be used to determine whether a given representation or its character is a smooth point in the representation or character variety, respectively.

4.3.2. Coboundaries at $p$-reps. First we determine the coboundaries $B^{1}:=B^{1}\left(W_{p / q} ; \mathfrak{s l}_{2}^{\rho}\right)$ for a p-rep in partially-diagonal normal form $\rho \in \mathfrak{D}^{ \pm}\left(W_{p / q}\right)$. As in section 4.2, we will restrict ourselves to the case $\rho \in \mathfrak{D}^{+}$, as the negative trace case proceeds analogously.

Let

$$
e_{1}=\left(\begin{array}{ll}
0 & 1 \\
0 & 0
\end{array}\right), \quad e_{2}=\left(\begin{array}{cc}
1 & 0 \\
0 & -1
\end{array}\right) \quad \text { and } \quad e_{3}=\left(\begin{array}{ll}
0 & 0 \\
1 & 0
\end{array}\right)
$$

be a basis for the Lie algebra $\mathfrak{s l}_{2}:=\mathfrak{s l}_{2}(\mathbb{C})$. For a matrix $A \in \mathfrak{s l}_{2}$, let $u_{A} \in B^{1}$ be the 1-coboundary defined by $u_{A}(\gamma)=A-\gamma \cdot A$ for $\gamma \in \pi_{1}\left(W_{p / q}\right)$. Given a representation $\rho \in \mathfrak{D}^{+}\left(W_{p / q}\right)$, the coboundaries are then generated by

$$
\begin{array}{llll}
u_{e_{1}}: & \mu_{0} \mapsto\left(\begin{array}{cc}
0 & 1-s^{2} \\
0 & 0
\end{array}\right), & \mu_{1} \mapsto\left(\begin{array}{cc}
a & 1-a^{2} \\
1 & -a
\end{array}\right), \\
u_{e_{2}}: & \mu_{0} \mapsto\left(\begin{array}{cc}
0 & 0 \\
0 & 0
\end{array}\right), & \mu_{1} \mapsto\left(\begin{array}{cc}
2(a-1)^{2} & -2 a(a-1)^{2} \\
2(a-2) & -2(a-1)^{2}
\end{array}\right), \\
u_{e_{3}}: & \mu_{0} \mapsto\left(\begin{array}{ccc}
0 & 0 \\
1-s^{-2} & 0
\end{array}\right), & \mu_{1} \mapsto\left(\begin{array}{cc}
(2-a)(a-1)^{2} & (a-1)^{4} \\
-(a-1)(a-3) & -(2-a)(a-1)^{2}
\end{array}\right) .
\end{array}
$$

Since cochains are determined by their images on the generators, we will implicitly use the isomorphism $\tau: \mathfrak{s l}_{2}^{2} \rightarrow \mathbb{C}^{6}$ given by

$$
\left(\left(\begin{array}{cc}
x_{1} & x_{2} \\
x_{3} & -x_{1}
\end{array}\right),\left(\begin{array}{cc}
y_{1} & y_{2} \\
y_{3} & -y_{1}
\end{array}\right)\right) \mapsto\left(x_{1}, x_{2}, x_{3}, y_{1}, y_{2}, y_{3}\right)
$$

to identify a cochain $u$ with an element of $\mathbb{C}^{6}$. We can therefore think of $Z^{1}:=Z^{1}\left(W_{p / q} ; \mathfrak{s i}_{2}^{\rho}\right)$ and $B^{1}$ as subspaces of $\mathbb{C}^{6}$. 
All coboundaries are now of the form $\alpha_{1} u_{e_{1}}+\alpha_{2} u_{e_{2}}+\alpha_{3} u_{e_{3}}$ with $\alpha_{i} \in \mathbb{C}$. Seen as elements of $\mathbb{C}^{6}$, they hence lie in the span of the row vectors of the matrix

$$
\mathcal{B}:=\left(\begin{array}{cccccc}
0 & 1-s^{2} & 0 & a & 1-a^{2} & 1 \\
0 & 0 & 0 & 2(a-1)^{2} & -2 a(a-1)^{2} & 2(a-2) \\
0 & 0 & 1-s^{-2} & (2-a)(a-1)^{2} & (a-1)^{4} & -(a-1)(a-3)
\end{array}\right) .
$$

Since $s \neq \pm 1$, one sees that the rank of this matrix, and hence the dimension of the subspace of coboundaries, is 3 .

Note that this remains true when $\rho$ is reducible, as then $a=1$ and $\mathcal{B}$ becomes

$$
\mathcal{B}^{\text {red }}=\left(\begin{array}{cccccc}
0 & 1-s^{2} & 0 & 1 & 0 & 1 \\
0 & 0 & 0 & 0 & 0 & -2 \\
0 & 0 & 1-s^{-2} & 0 & 0 & 0
\end{array}\right)
$$

4.3.3. Cocycle condition from the first relation. Let $\left(B^{1}\right)^{\perp}$ be the orthogonal complement of the subspace of coboundaries $B^{1}$ in $\mathbb{C}^{6}$ with respect to the standard inner product. For each cohomology element $\bar{u} \in H^{1}$ there then is a unique representative $u \in\left(B^{1}\right)^{\perp}$ such that $\bar{u}=u+B^{1}$. The orthogonal complement is given by the kernel of the matrix $\mathcal{B}$, and we can therefore identify $H^{1}$ with a subspace of $\left(B^{1}\right)^{\perp}$ in $\mathbb{C}^{6}$. In particular, we can obtain it as the kernel of the linear map given by the matrix $\mathcal{B}$ adjoined with additional rows corresponding to the cocycle versions of the group relations.

We therefore examine the effect of the first group relation on cocycles: Using $w_{1}:=$ $\mu_{1} \mu_{0} \mu_{1} \mu_{0}^{-1} \mu_{1}^{-1} \mu_{0}^{-1} \mu_{1} \mu_{0}=\mu_{0} \mu_{1} \mu_{0}^{-1} \mu_{1}^{-1} \mu_{0}^{-1} \mu_{1} \mu_{0} \mu_{1}=: w_{2}$, we see that each cocycle $u \in Z^{1}$ has to satisfy the matrix equation $u\left(w_{1}\right)-u\left(w_{2}\right)=0$. Assuming that $u\left(\mu_{0}\right)=\left(\begin{array}{cc}x_{1} & x_{2} \\ x_{3} & -x_{1}\end{array}\right)$ and $u\left(\mu_{1}\right)=\left(\begin{array}{cc}y_{1} & y_{2} \\ y_{3} & -y_{1}\end{array}\right)$ as in (43), we can iteratively use the cocycle condition (38) to expand this expression, yielding a matrix with entries which are polynomials in $x_{1}, x_{2}$, $x_{3}, y_{1}, y_{2}$ and $y_{3}$ with coefficients in $\mathbb{C}\left[a^{ \pm 1}, s^{ \pm 1}\right]$.

When $\rho$ is reducible (but non-abelian), we find that only the lower left entry of $u\left(w_{1}\right)=u\left(w_{2}\right)$ gives a non-vanishing equation, which we write in vector form as

$$
\left(\begin{array}{c}
0 \\
-2\left(s^{2}-1\right) \\
0 \\
-2\left(s^{2}-1\right)^{2} \\
s^{-2}\left(s^{2}-1\right)^{2}\left(s^{4}-s^{2}-1\right) \\
0
\end{array}\right)^{T}\left(\begin{array}{l}
x_{1} \\
x_{2} \\
x_{3} \\
y_{1} \\
y_{2} \\
y_{3}
\end{array}\right)=0 .
$$

This vector can now easily be seen to be linearly independent from the row vectors in the matrix $\mathcal{B}^{\text {red }}$ in (45).

On the other hand, when $\rho$ is irreducible (and hence $a \neq 1$ ), the matrix obtained is far more complicated. After dividing the entries by a common nonzero factor of $s^{-8}\left(s^{2}-\right.$ $1)(a-1)$ and reducing all coefficient polynomials in $\mathbb{C}\left[a^{ \pm 1}, s^{ \pm 1}\right]$ modulo $r_{1}^{+}$, one finds that the upper left entry yields the equation

$$
v^{T}\left(\begin{array}{llllll}
x_{1} & x_{2} & x_{3} & y_{1} & y_{2} & y_{3}
\end{array}\right)^{T}=0,
$$

where the vector $v$ is given by

$$
\left(\begin{array}{c}
-4\left(a^{3} s^{2}-a^{3}-7 a^{2} s^{2}+7 a^{2}+2 a s^{4}+8 a s^{2}-14 a+4 s^{2}+8\right) \\
-2\left(a^{2} s^{2}-a^{2}-2 a s^{4}-a s^{2}+3 a+s^{4}-2 s^{2}-2\right) \\
2\left(a^{4} s^{2}-a^{4}-9 a^{3} s^{2}+9 a^{3}+24 a^{2} s^{2}-29 a^{2}-a s^{6}-16 a s^{2}+39 a+s^{6}-2 s^{4}-8 s^{2}-18\right) \\
2 s^{2}\left(-6 a s^{2}+3 a^{2} s^{2}-a s^{4}-a^{2}+3 a-2\right) \\
a s^{6}+2 a s^{4}-2 a s^{2}+a-2 s^{4}+4 s^{2}-2 \\
-3 a^{3} s^{2}+a^{3}+16 a^{2} s^{2}-8 a^{2}-4 a s^{4}-19 a s^{2}+17 a-2 s^{2}-10
\end{array}\right)
$$

and $v^{T}$ denotes the transposed vector. Denote the first coefficient of $v$ (corresponding to $x_{1}$ ) by $\psi_{1}$. One way to see that $v$ is linearly independent from the row vectors in the matrix $\mathcal{B}$ in (44) is to verify that $\psi_{1}$ does not vanish at a representation $\rho \in \mathfrak{D}^{+}\left(W_{p / q}\right)$ : 
Using the computer algebra system Maple ${ }^{\mathrm{TM}}$ (Map) to calculate a reduced Groebner basis with respect to the pure lexicographic order $a>s$ for the ideal generated by $\psi_{1}$ and $r_{1}^{+}$, we find that its first generator is a power of $s$, implying that the only common zeros with $r_{1}^{+}$lie in the subspace defined by $s=0$, a contradiction to our assumptions.

We remark that the other entries of $u\left(w_{1}\right)-u\left(w_{2}\right)$ yield equations which can be shown to be consequences of the coboundary conditions and equation (48).

4.3.4. Cocycle condition from the filling relation. We now study the condition forced on the cocycles after applying $p / q$-filling on the first cusp. Letting $u\left(\mu_{0}\right)=\left(\begin{array}{cc}x_{1} & x_{2} \\ x_{3} & -x_{1}\end{array}\right)$ and $u\left(\mu_{1}\right)=\left(\begin{array}{cc}y_{1} & y_{2} \\ y_{3} & -y_{1}\end{array}\right)$ as before, we first determine the image $u\left(\lambda_{0}\right)=u\left(\mu_{1} \mu_{0} \mu_{1}^{-1} \mu_{0}^{-1} \mu_{1}^{-1} \mu_{0} \mu_{1} \mu_{0}^{-1}\right)$. A direct calculation shows that

$$
u\left(\lambda_{0}\right)=:\left(\begin{array}{cc}
l_{1} & l_{2} \\
l_{3} & -l_{1}
\end{array}\right),
$$

where the normalized entries $l_{1}, l_{2}$ and $l_{3}$ are given by

$$
\begin{aligned}
l_{1}= & -4 s^{-6}\left(a^{3} s^{2}-a^{3}-2 a^{2} s^{2}+4 a^{2}-a s^{6}-2 a s^{4}-a s^{2}-5 a+s^{6}+2 s^{4}+2 s^{2}+2\right) \\
& -2 s^{-8}\left(a^{2} s^{2}-a^{2}-a s^{2}+3 a-2 s^{6}-2 s^{4}-2 s^{2}-2\right) \\
& +2 s^{-4}\left(a^{4} s^{2}-a^{4}-3 a^{3} s^{2}+5 a^{3}-3 a^{2} s^{2}-7 a^{2}+2 a s^{4}\right. \\
& \left.\quad+11 a s^{2}+a-2 s^{4}-2 s^{2}+2\right) \\
& -4 s^{-2}\left(a^{3} s^{2}-a^{3}-3 a^{2} s^{2}+4 a^{2}+2 a s^{2}-5 a+2\right) \\
& -s^{-4}\left(2 a^{2} s^{2}-2 a^{2}-a s^{6}-3 a s^{2}+6 a-4 s^{4}-2 s^{2}-4\right) \\
& +2 s^{-6}\left(a^{4} s^{2}-a^{4}-6 a^{3} s^{2}+7 a^{3}+12 a^{2} s^{2}-19 a^{2}\right. \\
& \left.\quad-5 a s^{2}+23 a-2 s^{4}-6 s^{2}-10\right) \\
& \quad \\
& \quad-4 s^{-6}\left(a^{4} s^{2}-a^{4}-a^{3} s^{2}+3 a^{3}-11 a^{2} s^{2}+3 a^{2}+17 a s^{2}-15 a+2 s^{2}+10\right) \\
& +s^{-8}\left(2 a^{3} s^{2}-2 a^{3}+4 a^{2}-15 a s^{4}+a-s^{4}-8 s^{2}-3\right) \\
& -s^{-4}\left(2 a^{5} s^{2}-2 a^{5}-4 a^{4} s^{2}+8 a^{4}-12 a^{3} s^{2}-6 a^{3}+32 a^{2} s^{2}-12 a^{2}\right. \\
& \left.\quad s^{4}-9\right) \\
& +2 s^{-2}\left(2 a^{3} s^{2}-2 a^{3}-5 a^{2} s^{2}+7 a^{2}+2 a s^{2}-8 a+s^{2}+3\right) a \\
& +s^{-4}\left(2 a^{3} s^{2}-2 a^{3}-5 a^{2} s^{2}+7 a^{2}-5 a s^{4}+2 a s^{2}-7 a+s^{4}-s^{2}+2\right) \\
& -s^{-6}\left(2 a^{5} s^{2}-2 a^{5}-11 a^{4} s^{2}+13 a^{4}+18 a^{3} s^{2}-32 a^{3}+4 a^{2} s^{2}\right. \\
& \left.\quad+30 a^{2}-28 a s^{2}+2 a-s^{2}-11\right)
\end{aligned}
$$

and

$$
\begin{aligned}
l_{3}= & -4 s^{-6}\left(a^{2} s^{2}-a^{2}-2 a s^{8}+2 a s^{6}-a s^{2}+3 a+2 s^{8}-6 s^{6}-2 s^{4}-2 s^{2}-2\right) \\
& +s^{-2}\left(a s^{6}-6 a s^{4}+7 a s^{2}-2 a-s^{6}+8 s^{4}-15 s^{2}+4\right) \\
& +s^{-4}\left(2 a^{3} s^{2}-2 a^{3}-8 a^{2} s^{2}+12 a^{2}+a s^{12}-6 a s^{10}+11 a s^{8}-4 a s^{6}-4 a s^{4}\right. \\
& \left.\quad+2 a s^{2}-22 a-s^{12}+8 s^{10}-19 s^{8}+12 s^{6}+12 s^{4}+12 s^{2}+12\right) \\
& -2 s^{-2}\left(2 a^{2} s^{2}-2 a^{2}-2 a s^{4}-3 a s^{2}+7 a+2 s^{4}-2 s^{2}-6\right) \\
& +s^{-4}\left(a s^{10}-7 a s^{8}+13 a s^{6}-12 a s^{4}+6 a s^{2}-a-s^{10}\right. \\
& \left.+9 s^{8}-22 s^{6}+22 s^{4}-12 s^{2}+2\right) \\
& +s^{-6}\left(2 a^{3} s^{2}-2 a^{3}-13 a^{2} s^{2}+15 a^{2}+a s^{8}+5 a s^{6}+11 a s^{2}\right. \\
& \left.-31 a-s^{8}-3 s^{6}+10 s^{4}+14 s^{2}+18\right) .
\end{aligned}
$$


The relation $\mu_{0}^{p} \lambda_{0}^{q}=1$ then translates to $u\left(\mu_{0}^{p} \lambda_{0}^{q}\right)=\left(\begin{array}{ll}0 & 0 \\ 0 & 0\end{array}\right)$, where

$$
\begin{aligned}
& u\left(\mu_{0}^{p} \lambda_{0}^{q}\right)=u\left(\mu_{0}^{p}\right)+\mu_{0}^{p} \cdot u\left(\lambda_{0}^{q}\right)= \\
& =\left(1+\mu_{0}+\cdots+\mu_{0}^{p-1}\right) \cdot u\left(\mu_{0}\right)+\mu_{0}^{p}\left(1+\lambda_{0}+\cdots+\lambda_{0}^{q-1}\right) \cdot u\left(\lambda_{0}\right) \\
& =\left(\begin{array}{cc}
p x_{1}+q l_{1} & x_{2} \sum_{k=0}^{p-1} s^{2 k}+s^{2 p} l_{2} \sum_{j=0}^{q-1} t^{2 j} \\
x_{3} \sum_{k=0}^{p-1} s^{-2 k}+s^{-2 p} l_{3} \sum_{j=0}^{q-1} t^{-2 j} & -p x_{1}-q l_{1}
\end{array}\right) .
\end{aligned}
$$

We remark that this computation is the reason for our choice of the parametrization in section 4.2 as we could otherwise not have arrived at a general form in terms of $p$ and $q$.

Now $s \neq \pm 1$ implies that we can use

$$
\sum_{k=0}^{p-1} s^{2 k}=\frac{s^{2 p}-1}{s^{2}-1}
$$

and

$$
\sum_{k=0}^{p-1} s^{-2 k}=s^{-2(p-1)} \sum_{k=0}^{p-1} s^{2 k}=s^{-2(p-1)} \frac{s^{2 p}-1}{s^{2}-1} .
$$

We now need to distinguish between the reducible and irreducible case: When $\rho$ is reducible, $a=1$ implies $t=1$ and hence $\sum_{j=0}^{q-1} t^{2 j}=q$. But then $s^{p} t^{q}=1$ also implies $s^{p}=1$, and equation (53) becomes

$$
\left(\begin{array}{ll}
0 & 0 \\
0 & 0
\end{array}\right)=\left(\begin{array}{cc}
p x_{1}+q l_{1} & q l_{2} \\
q l_{3} & -p x_{1}-q l_{1}
\end{array}\right)
$$

But note that $a=1$ implies that $l_{1}=\left(s^{2}-s^{-2}\right) y_{2}, l_{2}=0$ and $l_{3}=-2 s^{-2} x_{2}+2\left(s^{-2}-\right.$ 1) $y_{1}+\left(s^{-4}-2+s^{2}\right) y_{2}$. It is then easy to see that $q l_{3}=0$ is already a consequence of the coboundary conditions, and so the only new condition remaining from (56) is $(p / q) x_{1}+l_{1}=0$. Adjoining this condition in row vector form together with the vector corresponding to equation (46) to the matrix from (45), we then obtain a presentation matrix for the cohomology group $H^{1}\left(W_{p / q} ; \mathfrak{s l}_{2}^{\rho}\right)$, viewed as a subspace of $\mathbb{C}^{6}$ :

$$
\left(\begin{array}{cccccc}
0 & 1-s^{2} & 0 & 1 & 0 & 1 \\
0 & 0 & 0 & 0 & 0 & -2 \\
0 & 0 & 1-s^{2} & 0 & 0 & 0 \\
0 & 2\left(1-s^{2}\right) & 0 & -2\left(1-s^{2}\right)^{2} & s^{-2}\left(s^{4}-s^{2}-1\right)\left(1-s^{2}\right)^{2} & 0 \\
p / q & 0 & 0 & 0 & s^{-2}\left(s^{4}-1\right) & 0
\end{array}\right) .
$$

The rank of this matrix can easily be seen to be 5 for all $s \neq \pm 1$, and we therefore obtain

$$
\operatorname{dim}_{\mathbb{C}} H^{1}\left(W_{p / q} ; \mathfrak{s l}_{2}^{\rho}\right)=1 .
$$

On the other hand, when $\rho$ is irreducible, we can use $t \neq \pm 1$ to rewrite (53) as

$$
\begin{aligned}
u\left(\mu_{0}^{p} \lambda^{q}\right) & =\left(\begin{array}{cc}
p x_{1}+q l_{1} & \frac{s^{2 p}-1}{s^{2}-1} x_{2}+s^{2 p} \frac{t^{2 q}-1}{t^{2}-1} l_{2} \\
s^{-2(p-1)} \frac{s^{2 p}-1}{s^{2}-1} x_{3}+s^{-2 p} t^{-2(q-1)} \frac{t^{2 q}-1}{t^{2}-1} l_{3} & -p x_{1}-q l_{1}
\end{array}\right) \\
& =\left(\begin{array}{cc}
p x_{1}+q l_{1} & \frac{s^{2 p}-1}{s^{2}-1} x_{2}+\frac{1-s^{2 p}}{t^{2}-1} l_{2} \\
s^{2} \frac{1-t^{2 q}}{s^{2}-1} x_{3}-t^{2} \frac{1-t^{2 q}}{t^{2}-1} l_{3} & -p x_{1}-q l_{1}
\end{array}\right) \\
& =\left(\begin{array}{cc}
p x_{1}+q l_{1} & \left(s^{2 p}-1\right)\left(\frac{1}{s^{2}-1} x_{2}-\frac{1}{t^{2}-1} l_{2}\right) \\
s^{-2 p}\left(s^{2 p}-1\right)\left(\frac{s^{2}}{s^{2}-1} x_{3}-\frac{t^{2}}{t^{2}-1} l_{3}\right) & -p x_{1}-q l_{1}
\end{array}\right) .
\end{aligned}
$$

From the upper right and lower left entries of this matrix we now see that either $s^{2 p}=1$ (which we know cannot be true by Lemma13, since $s$ is also a root of $\operatorname{res}_{p, q}$ ), or $\left(t^{2}-1\right) x_{2}=$ $\left(s^{2}-1\right) l_{2}$ and $s^{2}\left(t^{2}-1\right) x_{3}=t^{2}\left(s^{2}-1\right) l_{3}$. After some careful algebraic manipulations, it can be verified that these last two conditions are in fact consequences of the coboundary conditions (44) and equation (48). The only new information comes therefore from the 
upper left matrix entry. Since $q>0$, we can rewrite this in the form $l_{1}+(p / q) x_{1}=0$, which gives us (after normalizing with respect to $r_{1}^{+}$) the condition

$$
w^{T}\left(\begin{array}{llllll}
x_{1} & x_{2} & x_{3} & y_{1} & y_{2} & y_{3}
\end{array}\right)^{T}=0,
$$

where the vector $w$ is given by

$$
\left(\begin{array}{c}
p / q-4 s^{-6}\left(a^{3} s^{2}-a^{3}-2 a^{2} s^{2}+4 a^{2}-a s^{6}-2 a s^{4}-a s^{2}-5 a+s^{6}+2 s^{4}+2 s^{2}+2\right) \\
-2 s^{-8}\left(a^{2} s^{2}-a^{2}-a s^{2}+3 a-2 s^{6}-2 s^{4}-2 s^{2}-2\right) \\
2 s^{-4}\left(a^{4} s^{2}-a^{4}-3 a^{3} s^{2}+5 a^{3}-3 a^{2} s^{2}-7 a^{2}+2 a s^{4}+11 a s^{2}+a-2 s^{4}-2 s^{2}+2\right) \\
-4 s^{-2}\left(a^{3} s^{2}-a^{3}-3 a^{2} s^{2}+4 a^{2}+2 a s^{2}-5 a+2\right) \\
-s^{-4}\left(2 a^{2} s^{2}-2 a^{2}-a s^{6}-3 a s^{2}+6 a-4 s^{4}-2 s^{2}-4\right) \\
2 s^{-6}\left(a^{4} s^{2}-a^{4}-6 a^{3} s^{2}+7 a^{3}+12 a^{2} s^{2}-19 a^{2}-5 a s^{2}+23 a-2 s^{4}-6 s^{2}-10\right)
\end{array}\right) .
$$

Adjoining the two row vectors corresponding to equations (48) and (61) to the matrix $B$, we now obtain a presentation matrix for $H^{1}\left(W_{p / q} ; \mathfrak{s}_{2}^{\rho}\right)$. With some care and making sure no polynomial used in division can be zero on $\mathfrak{D}^{+}$, we can row-reduce this matrix to the form

$$
\left(\begin{array}{cccccc}
\psi_{1} & 0 & 0 & 0 & * & * \\
0 & s^{2}-1 & 0 & 0 & -1 & -(a-1)^{-2} \\
0 & 0 & s^{-2}-1 & 0 & s^{2}(a-1)^{2} & s^{2} \\
0 & 0 & 0 & (a-1)^{2} & -a(a-1)^{2} & a-2 \\
0 & 0 & 0 & 0 & * & *
\end{array}\right)
$$

where each $*$ denotes a polynomial in $a$ and $s$ (normalized modulo $r_{1}^{+}$) which does not vanish on $\mathfrak{D}^{+}$, and $\psi_{1}$ is the first coefficient of the vector in equation (48). This shows that the rank of this matrix is 5 , and we therefore find that

$$
\operatorname{dim}_{\mathbb{C}} H^{1}\left(W_{p / q} ; \mathfrak{s l}_{2}^{\rho}\right)=1
$$

or equivalently,

$$
\operatorname{dim}_{\mathbb{C}} Z^{1}\left(W_{p / q} ; \mathfrak{s l}_{2}^{\rho}\right)=4 .
$$

Since Corollary [19] showed that a non-abelian reducible p-rep lies on a non-trivial curve in $\mathfrak{R}\left(W_{p / q}\right)$, and an irreducible p-rep does so automatically, Theorem A of Boy02 now shows:

Proposition 22. The character $\chi_{\rho}$ of a (reducible or irreducible) p-prep $\rho \in \mathfrak{R}\left(W_{p / q}\right)$ is a smooth point of $\mathfrak{X}^{i r r}(W)$ and is contained in a unique non-trivial curve. Furthermore, we have $T_{\chi \rho}^{Z a r}\left(\mathfrak{X}\left(W_{p / q}\right)\right) \cong H^{1}\left(W_{p / q} ; \mathfrak{s}_{2}^{\rho}\right)$, presented as a subset of $\mathbb{C}^{6}$ as the kernel of the matrix (57) in the reducible, and the matrix (62) in the irreducible case.

This then concludes the proof of Proposition 16, and furthermore provides an explicit parametrization for non-trivial cocycles in $Z^{1}\left(M ; \mathfrak{s l}_{2}^{\rho}\right)$ at a given representation $\rho \in \mathfrak{D}^{+}$. This will be needed in the following section, when we will study deformations of these representations.

4.4. Deformations of representations and degree of the trace function. We now turn our attention to the proof of Proposition 17.

Since Proposition 22 showed that $\mathfrak{X}^{\mathrm{irr}}\left(W_{p / q}\right)$ is smooth and positive-dimensional at the character of a p-rep $\rho$, we can use the existence of non-trivial cocycles to find a smooth deformation $\rho_{\nu}$ of $\rho_{0}=\rho$, which admits an expansion of the form

$$
\rho_{\nu}=\exp \left(\nu u_{1}+\nu^{2} u_{2}+\nu^{3} u_{3}+\ldots\right) \rho
$$

where $u_{j}: \pi_{1}(G) \rightarrow \mathfrak{s l}_{2}(\mathbb{C})$, and $u_{1} \in Z^{1}\left(G ; \mathfrak{s l}_{2}^{\rho}\right)$ (see e.g. [HPSP01, $\S$ ] or [Gol84]).

Developing $f_{\chi_{\rho_{\nu}}}=\left(\operatorname{trace} \rho_{\nu}(\gamma)\right)^{2}-4$ using the expansion

$$
\exp (A)=\sum_{j=1}^{\infty} A^{j} / j !
$$


for $A \in \mathfrak{s l}_{2}(\mathbb{C})$, we find that the trace function $f_{\mu_{1}}\left(\chi_{\rho_{\nu}}\right)$ has the following Taylor expansion at $\rho_{0}$ (see e.g. Lemma 1.3 in [BAB01]):

$$
f_{\mu_{1}}\left(\chi_{\rho_{\nu}}\right)=f_{\mu_{1}}\left(\chi_{\rho_{0}}\right)+2 I_{\mu_{1}}\left(\chi_{\rho_{0}}\right) \operatorname{trace}\left(u_{1}\left(\mu_{1}\right) \rho_{0}\left(\mu_{1}\right)\right) \nu+O\left(\nu^{2}\right) .
$$

Note that in our case $G:=\pi_{1}\left(W_{p / q}\right)$ and $I_{\mu_{1}}\left(\chi_{\rho_{0}}\right)=\left(\text { trace } \rho_{0}\left(\mu_{1}\right)\right)^{2}=4$ for $\rho_{0} \in \mathfrak{D}^{ \pm}$. Since $f_{\mu_{1}}\left(\chi_{\rho_{0}}\right)=0$ at a p-rep $\rho_{0}$, the order of this zero is 1 iff there is a non-trivial cocycle $u_{1} \in Z^{1}\left(W_{p / q} ; \mathfrak{s l}_{2}^{\rho}\right)$ satisfying

$$
\operatorname{trace}\left(u_{1}\left(\mu_{1}\right) \rho_{0}\left(\mu_{1}\right)\right) \neq 0 .
$$

To prove this, note that for $\rho_{0} \in \mathfrak{D}^{+}$and an arbitrary cochain $u_{1}$ we have

$$
\begin{aligned}
\operatorname{trace}\left(u_{1}\left(\mu_{1}\right) \rho_{0}\left(\mu_{1}\right)\right) & =\operatorname{trace}\left(\begin{array}{cc}
y_{1} & y_{2} \\
y_{3} & -y_{1}
\end{array}\right)\left(\begin{array}{cc}
a & -(a-1)^{2} \\
1 & 2-a
\end{array}\right) \\
& =2(a-1) y_{1}+y_{2}-(a-1)^{2} y_{3},
\end{aligned}
$$

and an analogous expression for $\rho_{0} \in \mathfrak{D}^{-}$.

Let $P$ be the matrix obtained by adjoining the row vector corresponding to this expression to the presentation matrix for $H^{1}\left(W_{p / q} ; \mathfrak{s l}_{2}^{\rho}\right)$ from (57) or (62), respectively. The existence of a non-trivial cocycle $u_{1}$ such that (69) is equal to a non-zero value $c \in \mathbb{C}^{*}$ is now equivalent to finding a solution $u_{1}:=\left(x_{1}, x_{2}, x_{3}, y_{1}, y_{2}, y_{3}\right)$ to the linear equation system

$$
P\left(x_{1}, x_{2}, x_{3}, y_{1}, y_{2}, y_{3}\right)^{T}=(0,0,0,0,0, c)^{T}
$$

and hence a non-trivial element in $H^{1}\left(W_{p / q}\right)$ represented by a cocycle $u_{1}$ which satisfies (68). In particular, if we can show that $\operatorname{det} P \neq 0$, the system (70) will in fact have a unique solution $u_{1}$ for any given $c \in \mathbb{C}^{*}$.

Again restricting attention to $\rho_{0} \in \mathfrak{D}^{+}$, we will first discuss the case when $\rho_{0}$ is reducible: In this case, the matrix $P$ is given by

$$
P=\left(\begin{array}{cccccc}
0 & 1-s^{2} & 0 & 1 & 0 & 1 \\
0 & 0 & 0 & 0 & 0 & -2 \\
0 & 0 & 1-s^{2} & 0 & 0 & 0 \\
0 & 2\left(1-s^{2}\right) & 0 & -2\left(1-s^{2}\right)^{2} & s^{-2}\left(s^{4}-s^{2}-1\right)\left(1-s^{2}\right)^{2} & 0 \\
p / q & 0 & 0 & 0 & s^{-2}\left(s^{4}-1\right) & 0 \\
0 & 0 & 0 & 0 & 1 & 0
\end{array}\right),
$$

and we find $\operatorname{det} P=-(4 p / q)\left(1-s^{2}\right)^{2}\left(s^{4}-2 s^{2}+2\right)$. Now recall from section 3.3 that the eigenvalue $s$ of a reducible p-rep must satisfy $s^{p}=1, s \neq \pm 1$. None of the roots of $s^{4}-2 s^{2}+2=0$ lie on the unit circle, and so $\operatorname{det} P \neq 0$ unless $p=0$ : In that case, the trace in (69) will indeed be zero for all cocycles $u_{1}$, and hence the order of a zero of $f_{\mu_{1}}$ at the character of a reducible non-abelian p-rep is indeed greater than 1 . This also reflects the fact that there is a whole curve of non-abelian reducible p-reps for $s \in \mathbb{C}^{*}-\{ \pm 1\}$ in this case (since $p=0, q=1$ implies $s^{p} t^{q}=t=1$, and there are no further conditions on $s)$.

This proves Proposition 17 in the case of a non-abelian reducible p-rep, $p / q \neq 0$.

We will now discuss the remaining case, when $\rho_{0} \in \mathfrak{D}^{+}$is irreducible. Appending the row vector corresponding to the expression (69) to the matrix (62) and applying GaussJordan to reduce the matrix, we obtain the $6 \times 6$-matrix

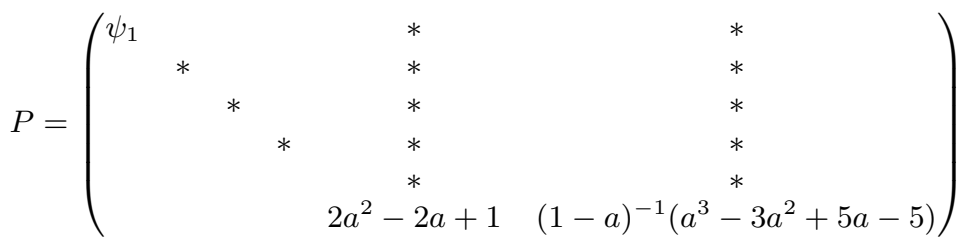


Denote by $d$ the determinant of the lower right $2 \times 2$-block of this matrix. We wish to show that this determinant is not zero for $\rho_{0} \in \mathfrak{D}^{+}$. To show this, we again used the computer algebra system Maple ${ }^{\mathrm{TM}}$ to calculate a Groebner basis of the ideal generated by $r_{1}^{+}$and the numerator of $d$ with respect to a lexicographic ordering with $a>s$ (we remark that this computation took more than 24 hours on a modern PC). We find that the first basis element is given by $s^{30} d_{1} d_{2}$ where

$$
d_{1}=p(p-4 q) s^{4}+\left(-6 p^{2}+24 p q-32 q^{2}\right) s^{2}+p(p-4 q)
$$

and

$$
\begin{aligned}
d_{2}= & 22 s^{40}-438 s^{38}+4185 s^{36}-24868 s^{34}+101875 s^{32}-304088 s^{30} \\
+ & 683740 s^{28}-1182928 s^{26}+1598312 s^{24}-1708564 s^{22}+1466502 s^{20} \\
& -1027864 s^{18}+595850 s^{16}-286072 s^{14}+115452 s^{12}-41808 s^{10} \\
& +14586 s^{8}-4582 s^{6}+1097 s^{4}-164 s^{2}+11 .
\end{aligned}
$$

If $d$ were to vanish at an irreducible representation $\rho_{0} \in \mathfrak{D}^{+}$, each of the polynomials in the Groebner basis, and in particular the first element, would have to vanish. We will therefore show that neither $d_{1}$ nor $d_{2}$ vanish at a representation $\rho_{0} \in \mathfrak{D}^{+}$, which shows that $d$ cannot vanish there. For this, we will make use of the fact that the parametrization of any irreducible representation $\rho_{0} \in \mathfrak{D}^{ \pm}$also satisfies $\operatorname{res}_{p, q}=0$ (as determined in section 3.4). It suffices therefore to show that $\operatorname{res}_{p, q}$ has no roots in common with $d_{1}$ and $d_{2}$.

As for $d_{1}$, we note that its four roots are

$$
\pm \frac{\left(\left(3(p-2 q)^{2}+4 q^{2}\right) \pm\left(2|p-2 q| \sqrt{2(p-2 q)^{2}+8 q^{2}}\right)\right)^{1 / 2}}{\sqrt{p(p-4 q)}}
$$

It is easy to see that in fact these roots are all real-valued when $p>4 q>0$ or when $p<0<4 q$, and all imaginary when $0<p<4 q$. To see this, simply note that

$$
\left(3(p-2 q)^{2}+4 q^{2}\right)^{2}-\left(2|p-2 q| \sqrt{2(p-2 q)^{2}+8 q^{2}}\right)^{2}=p^{2}(p-4 q)^{2} \geq 0
$$

which shows that the numerator in (75) is always real-valued.

But note that for $p>4 q>0$ or $p<0<4 q$, apart from \pm 1 , none of the non-zero roots of $\operatorname{res}_{p, q}$ are real-valued by Lemma 11 Similarly for $0<p<4 q$, none of its non-zero roots are pure imaginary by Lemma 12 So $d_{1}$ in fact does not share any roots with res ${ }_{p, q}$.

It now remains to be shown that the polynomial $d_{2}$ has no common roots with res $\operatorname{s}_{p, q}$. To see this, first observe that $d_{2}$ is an irreducible polynomial, while its leading coefficient is $22 \neq 1$. On the other hand, while $\operatorname{res}_{p, q}$ is in general not irreducible (consider for example $q=1$ and $p$ even), it is a monic polynomial with integer coefficients - and therefore so are its irreducible factors: The Chebyshev polynomial $T_{q}(y)$ has leading term $2^{q-1} y^{q}$, and substituting $y=-s^{2} / 2+2-s^{-2} / 2$ gives the leading term $1 / 2 s^{2 q}$. Now note that the "outer" terms of $\operatorname{res}_{p, q}$ are $s^{p-2 q}$ and $s^{-p+2 q}$, and the middle terms are given by $2 T_{q}(y)$.

We conclude that all roots of $\operatorname{res}_{p, q}$ are algebraic integers, while this is not true for any of the roots of $d_{2}$. This shows that $d_{2}$ and $\operatorname{res}_{p, q}$ have no roots in common. We show the 40 roots of $d_{2}$ in figure 3

We can therefore conclude that the $2 \times 2$ minor $d$ is indeed non-zero at characters of p-reps, and that hence $\operatorname{rank} P=6$ and $\operatorname{det} P \neq 0$, proving Proposition 17 for irreducible p-reps.

This then concludes the proof of Theorem 15, showing that the minimal seminorm $s$ is given by the number of characters of p-reps. 


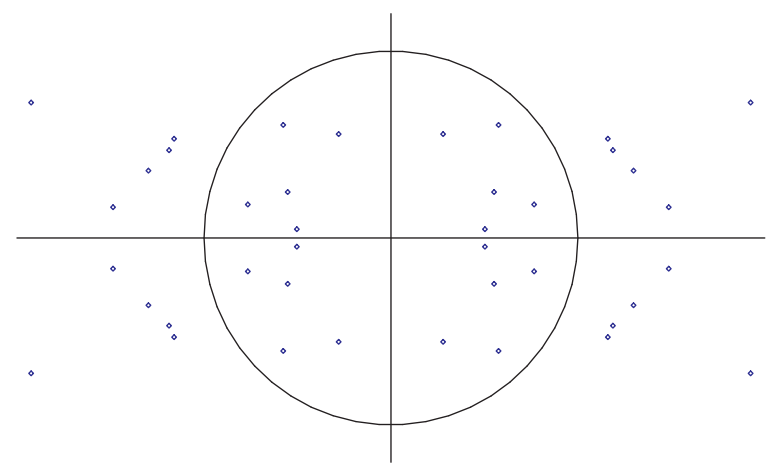

Figure 3 . The roots of the polynomial $d_{2}$

\section{Seifert fillings}

The goal of this section is to prove:

Proposition 23. For $p$ odd, the values of the total Culler-Shalen seminorm of the slopes 1,2 and 3 of $W_{p / q}$ are given by

$$
\begin{array}{ll}
\|1\|=s+2|p-6 q|-2 & (\text { if } p / q \neq 6), \\
\|2\|=s+3|p-4 q|-3 & (\text { if } p / q \neq 4), \\
\|3\|=s+4|p-3 q|-4 & (\text { if } p / q \neq 3),
\end{array}
$$

where $s$ is the minimal total Culler-Shalen seminorm.

Note that while $p / q \neq 6$ and $p / q \neq 4$ are already satisfied when $p$ is odd, we mention these conditions for completeness.

The proof is based on the well-known fact (see e.g. [MP06, § 9] or DT03]) that for $\sigma \in\{1,2,3\}$, the closed manifold $W_{p / q}(\sigma)=W(p / q, \sigma)$ is Seifert fibered with base orbifold a sphere with three cone points (such manifolds are often called small Seifert):

- $W(p / q, 1)$ is Seifert fibered over $S^{2}(2,3,|p-6 q|)$ if $p / q \neq 6$

- $W(p / q, 2)$ is Seifert fibered over $S^{2}(2,4,|p-4 q|)$ if $p / q \neq 4$

- $W(p / q, 3)$ is Seifert fibered over $S^{2}(3,3,|p-3 q|)$ if $p / q \neq 3$

This can be seen from the fact that $W(1), W(2)$ and $W(3)$ are all Seifert fibred with base orbifold a disc with two cone points: $D^{2}(2,3), D^{2}(2,4)$ and $D^{2}(3,3)$, respectively. Furthermore, the corresponding fibre slopes are 6,4 and 3, respectively. Therefore, applying a second Dehn filling generically produces a Seifert fibred manifold with base orbifold a sphere with three cone points, with only one exception each: When filling along the fibre slope, the resulting closed manifold is a connected sum of lens spaces: $W(1,6) \cong L_{2} \# L_{3}$, $W(2,4) \cong L_{2} \# L_{4}$ and $W(3,3) \cong L_{3} \# L_{3}$.

The methods from BAB01 now enable us to explictly determine the Culler-Shalen seminorm of these small Seifert manifolds:

Let $\sigma \in\{1,2,3\}$ be one of the Seifert filling slopes for $W_{p / q}$. Theorem C of [BAB01] shows that in this situation $\|\sigma\|=s+2 A$, where $A$ is the number of characters of nonabelian representations $\rho \in \mathfrak{R}\left(W_{p / q}\right)$ satisfying $\rho(\sigma)= \pm I$ whose characters lie on nontrivial curves in $\mathfrak{X}\left(W_{p / q}\right)$.

Note that such a representation necessarily induces a (non-abelian) $\mathrm{PSL}_{2}(\mathbb{C})$ representation $\bar{\rho} \in \bar{\Re}\left(W_{p / q}\right)$ with $\bar{\rho}(\sigma)= \pm I$, which in turn factors through to give a representation $\bar{\rho}^{\prime} \in \bar{\Re}\left(W_{p / q}(\sigma)\right)$. (An abelian irreducible representation $\bar{\rho} \in \bar{\Re}$ necessarily has image isomorphic to $\mathbb{Z} / 2 \oplus \mathbb{Z} / 2$, generated by $\pm\left(\begin{array}{cc}i & 0 \\ 0 & -i\end{array}\right)$ and $\pm\left(\begin{array}{cc}0 & -b \\ b^{-1} & 0\end{array}\right)$ for some $b \in \mathbb{C}^{*}$. This 
possibility is excluded in our situation by the fact that $p$ is odd.) Since $W_{p / q}(\sigma)$ has base orbifold $S^{2}(a, b, c)$, a sphere with three cone points, there is a presentation of $\pi_{1}\left(W_{p / q}(\sigma)\right)$ of the form

$$
\left.\pi_{1}\left(W_{p / q}(\sigma)\right)=\langle x, y, h| h \text { central, } x^{a}=h^{a^{\prime}}, y^{b}=h^{b^{\prime}},(x y)^{c}=h^{c^{\prime}}\right\rangle
$$

where $\operatorname{gcd}\left(a, a^{\prime}\right)=\operatorname{gcd}\left(b, b^{\prime}\right)=\operatorname{gcd}\left(c, c^{\prime}\right)=1($ cf. Jac80, $\left.\S \mathrm{VI}]\right)$.

Lemma 3.1 from BAB01 now shows that the representation $\bar{\rho}^{\prime} \in \bar{\Re}\left(W_{p / q}(\sigma)\right)$ again factors through to give a representation $\bar{\rho}^{\prime \prime} \in \overline{\mathfrak{R}}(\Delta(a, b, c))$, where $\Delta(a, b, c) \cong$ $\pi_{1}^{\text {orb }}\left(S^{2}(a, b, c)\right)$ is the $(a, b, c)$ triangle group with presentation

$$
\Delta(a, b, c)=\left\langle x, y \mid x^{a}=y^{b}=(x y)^{c}=1\right\rangle .
$$

Conversely, a given representation $\bar{\rho}^{\prime \prime} \in \overline{\mathfrak{R}}(\Delta(a, b, c))$ induces a unique representation $\bar{\rho}^{\prime} \in \bar{\Re}\left(W_{p / q}(\sigma)\right)$, which in turn induces a unique representation $\bar{\rho} \in \overline{\mathfrak{R}}\left(W_{p / q}\right)$ satisfying $\bar{\rho}(\sigma)= \pm I$. We summarize the situation in the following diagram:



Using the following theorem from BAB01, which we restate here in slightly adapted form, together with the fact that $H_{1}\left(W_{p / q}(r / s)\right)=\mathbb{Z} /|p| \oplus \mathbb{Z} /|r|$, one can now explicitly determine the number of $\mathrm{PSL}_{2}(\mathbb{C})$ characters of $\Delta(a, b, c)$, and hence of $\pi_{1}\left(W_{p / q}\right)$, for each of the Seifert filling slopes:

Theorem ( BAB01, Theorem D).

Let $M$ be a non-Haken small Seifert manifold, with base orbifold of the form $S^{2}(a, b, c)$ where $a, b, c \geq 2$.

(1) The number of reducible $\mathrm{PSL}_{2}(\mathbb{C})$-characters of $\pi_{1}(M)$ is

$$
\left\lfloor\frac{\left|H_{1}(M)\right|}{2}\right\rfloor+1+\left\{\begin{array}{ll}
0 & \text { if } \operatorname{gcd}(a, b, c) \text { is odd, } \\
1 & \text { if } \operatorname{gcd}(a, b, c) \text { is even. }
\end{array} .\right.
$$

(2) The number of $\mathrm{PSL}_{2}(\mathbb{C})$-characters of $\pi_{1}(M)$ representations with image a dihedral group of order at least 4 is

$$
\sigma(b, c)\left\lfloor\frac{a}{2}\right\rfloor+\sigma(a, c)\left\lfloor\frac{b}{2}\right\rfloor+\sigma(a, b)\left\lfloor\frac{c}{2}\right\rfloor-2 \sigma(a, b) \sigma(a, c) \sigma(b, c)
$$

where $\sigma(m, n)=\left\lfloor\frac{\operatorname{gcd}(2, m, n)}{2}\right\rfloor$ is 1 if $m$ and $n$ are both even, and 0 otherwise.

(3) The total number of $\mathrm{PSL}_{2}(\mathbb{C})$-characters of $\pi_{1}(M)$ is

$$
\begin{gathered}
\left\lfloor\frac{\left\lfloor H_{1}(M)\right\rfloor}{2}\right\rfloor+1+\left\lfloor\frac{a}{2}\right\rfloor\left\lfloor\frac{b}{2}\right\rfloor\left\lfloor\frac{c}{2}\right\rfloor+\left\lfloor\frac{a-1}{2}\right\rfloor\left\lfloor\frac{b-1}{2}\right\rfloor\left\lfloor\frac{c-1}{2}\right\rfloor \\
-\left\lfloor\frac{\operatorname{gcd}(a b, a c, b c)}{2}\right\rfloor+\left\lfloor\frac{\operatorname{gcd}(a, b)}{2}\right\rfloor+\left\lfloor\frac{\operatorname{gcd}(a, c)}{2}\right\rfloor+\left\lfloor\frac{\operatorname{gcd}(b, c)}{2}\right\rfloor
\end{gathered}
$$

The results are summarized in tables [6, 7 and 8 on page 29, Also included are the numbers of non-abelian reducible characters, which can be found by direct calculation. 


\begin{tabular}{c||c|cc|cc}
$\operatorname{gcd}(6, p)$ & total & irreducible & dihedral & reducible & non-ab. red. \\
\hline \hline 1 & $\frac{1}{2}(|p|+|p-6 q|)$ & $\frac{1}{2}(|p-6 q|-1)$ & 0 & $\frac{1}{2}(|p|+1)$ & 0 \\
3 & $\frac{1}{2}(|p|+|p-6 q|)$ & $\frac{1}{2}(|p-6 q|-1)$ & 0 & $\frac{1}{2}(|p|+1)$ & 0 \\
\hline 2 & $\frac{1}{2}(|p|+|p-6 q|)+1$ & $\frac{1}{2}|p-6 q|$ & 1 & $\frac{1}{2}|p|+1$ & 0 \\
6 & $\frac{1}{2}(|p|+|p-6 q|)$ & $\frac{1}{2}|p-6 q|-1$ & 1 & $\frac{1}{2}|p|+1$ & 1
\end{tabular}

TABLE 6. Number of $\mathrm{PSL}_{2}(\mathbb{C})$-characters of $\pi_{1} W_{p / q}(1)$

\begin{tabular}{c||c|cc|cc}
$\operatorname{gcd}(4, p)$ & total & irreducible & dihedral & reducible & non-ab. red. \\
\hline \hline 1 & $|p|+|p-4 q|$ & $|p-4 q|-1$ & $\frac{1}{2}(|p-4 q|-1)$ & $|p|+1$ & 0 \\
\hline 2 & $|p|+|p-4 q|+2$ & $|p-4 q|$ & $\frac{1}{2}|p-4 q|+1$ & $|p|+2$ & 0 \\
4 & $|p|+|p-4 q|+1$ & $|p-4 q|-1$ & $\frac{1}{2}|p-4 q|+1$ & $|p|+2$ & 1
\end{tabular}

TABLE 7. Number of $\mathrm{PSL}_{2}(\mathbb{C})$-characters of $\pi_{1} W_{p / q}(2)$

\begin{tabular}{c||c|cc|cc}
$\operatorname{gcd}(6, p)$ & total & irreducible & dihedral & reducible & non-ab. red. \\
\hline \hline 1 & $\frac{3}{2}(|p|-1)+|p-3 q|$ & $|p-3 q|-1$ & 0 & $\frac{3}{2}(|p|-1)+1$ & 0 \\
3 & $\frac{3}{2}(|p|-1)+|p-3 q|-1$ & $|p-3 q|-2$ & 0 & $\frac{3}{2}(|p|-1)+1$ & 1 \\
\hline 2 & $\frac{3}{2}|p|+|p-3 q|$ & $|p-3 q|-1$ & 0 & $\frac{3}{2}|p|+1$ & 0 \\
6 & $\frac{3}{2}|p|+|p-3 q|-1$ & $|p-3 q|-2$ & 0 & $\frac{3}{2}|p|+1$ & 1
\end{tabular}

TABLE 8. Number of $\mathrm{PSL}_{2}(\mathbb{C})$-characters of $\pi_{1} W_{p / q}(3)$ 
It now remains to determine the number of non-abelian $\mathrm{SL}_{2}(\mathbb{C})$ characters, i.e. to determine which of the $\mathrm{PSL}_{2}(\mathbb{C})$ representations $\bar{\rho} \in \overline{\mathfrak{R}}\left(W_{p / q}\right)$ lift to representations $\rho \in$ $\mathfrak{R}\left(W_{p / q}\right)$. There is in general an obstruction to this lifting, which is contained in the cohomology group

$$
H^{2}\left(W_{p / q} ; \mathbb{Z} / 2\right) \cong \begin{cases}0 & \text { if } p \text { is odd } \\ \mathbb{Z} / 2 & \text { if } p \text { is even. }\end{cases}
$$

When $p$ is even, we therefore possibly do have an obstruction to lifting, and it is to be expected that some representations do lift, but not others, making it impossible to directly make a statement about the number of $\mathrm{SL}_{2}(\mathbb{C})$ representations.

On the other hand, there is no obstruction to lifting when $p$ is odd, and we shall therefore restrict our attention to this case.

The number of lifts of a given $\mathrm{PSL}_{2}(\mathbb{C})$ representation is generically given by the cardinality of

$$
H^{1}\left(W_{p / q} ; \mathbb{Z} / 2\right) \cong \begin{cases}\mathbb{Z} / 2 & \text { if } p \text { is odd, } \\ \mathbb{Z} / 2 \oplus \mathbb{Z} / 2 & \text { if } p \text { is even, }\end{cases}
$$

but dihedral representations only lift half as many times as given by this number (see e.g. Lemma 5.5 of [BZ96] and the remarks following it for more details).

When $p$ is odd, dihedral $\mathrm{PSL}_{2}(\mathbb{C})$ characters are therefore covered by only one $\mathrm{SL}_{2}(\mathbb{C})$ character, whereas the remaining non-abelian characters are covered by two, giving us the results listed in table 9 . where the total number of non-abelian $\mathrm{SL}_{2}(\mathbb{C})$ characters shown in the last column gives the constant $A$ needed to conclude the proof of Proposition 23.

\begin{tabular}{c|ccc|c} 
& irred. non-dihedral & dihedral & non-ab. red. & total \\
\hline$W_{p / q}(1)$ & $|p-6 q|-1$ & 0 & 0 & $|p-6 q|-1$ \\
$W_{p / q}(2)$ & $|p-4 q|-1$ & $\frac{1}{2}(|p-4 q|-1)$ & 0 & $\frac{3}{2}(|p-4 q|-1)$ \\
$W_{p / q}(3)$ & $\left\{\begin{array}{lll}2|p-3 q|-2 & \text { if } 3 \nmid p & 0 \\
2|p-3 q|-4 & \text { if } 3 \mid p & 0\end{array}\right.$ & $\begin{cases}0 & \text { if } 3 \nmid p \\
2 & \text { if } 3 \mid p\end{cases}$ & $2(|p-3 q|-1)$
\end{tabular}

TABle 9. Number of non-abelian $\mathrm{SL}_{2}(\mathbb{C})$ characters for Seifert fillings of $W_{p / q}$ when $p$ is odd

\section{The total Culler-Shalen seminorm}

Putting together the results of the previous sections, we now completely determine the total Culler-Shalen seminorm of the manifolds $W_{p / q}$ for $p$ odd and $p / q \neq 3$, proving Theorem 1 In particular, we will show that the upper bounds established in Theorem 15 (cf. section 4) for the minimal total Culler-Shalen seminorm $s$ are in fact attained, which in turn shows Corollary 4

Using the possible boundary slopes $\beta_{j}$ from section 2.2 we apply equation (4) with unknown coefficients $a_{j}$ and $s$ to the Seifert filling slopes 1, 2, 3 and compare with the explicit values (relative to $s$ ) obtained in Proposition 23

From these equations, we then obtain a linear system which we can try to solve for the unknown coefficients $a_{j}$ and $s$. Note that depending on the range of the filling slope $p / q$, the parametrization of the boundary slope $\beta_{2}$ changes (cf. table 3 on page 7), and hence we get different distances and accordingly different linear systems for each range. We will therefore discuss each range in sequence.

While there are some cases (when $p / q \in(3,4)$ or $p / q \in(4,6)$ ) when the linear system already determines a unique solution, the system is in general underdetermined and we need more information. This is where we use the upper bound for the total minimal 
seminorm $s$ established in Theorem [15] In two sub-cases $(p / q \in(2,3)$ and $p / q>6)$, it turns out that even this is not yet sufficient, but using a symmetry argument about the resultant $\operatorname{res}_{p, q}$, we can reduce the case to one which was already proven before.

As before, we will always assume $q>0$ and $p \in \mathbb{Z}$ with $(p, q)=1$ and (due to the restrictions from section 5) $p$ odd as well as $p / q \neq 3$.

6.1. Assuming $p / q \in(-\infty, 0), p$ odd. In this case, we obtain the following distances between the boundary, Seifert and meridional slopes:

\begin{tabular}{c|ccccccc}
$\Delta$ & $\beta_{1}=4$ & $\beta_{2}=\frac{4 q}{p}$ & $\beta_{3}=0$ & 1 & 2 & 3 & $\mu=\infty$ \\
\hline$\beta_{1}$ & 0 & $4(-p+q)$ & 4 & 3 & 2 & 1 & 1 \\
$\beta_{2}$ & & 0 & $4 q$ & $-p+4 q$ & $-2 p+4 q$ & $-3 p+4 q$ & $-p$ \\
$\beta_{3}$ & & & 0 & 1 & 2 & 3 & 1
\end{tabular}

TABLE 10. Distances between slopes in $W_{p / q}$ for $p / q \in(-\infty, 0), p$ odd, $q>0$

Using the expressions of the seminorms for the Seifert slopes from Proposition 23 in terms of the minimal seminorm $s$ on one hand and the corresponding linear combinations with the slope distances on the other, we get the following linear system to solve for $s, a_{1}$, $a_{2}$ and $a_{3}$ :

$$
\begin{aligned}
s+2(-p+6 q-1) & =\|1\|=3 a_{1}+(-p+4 q) a_{2}+a_{3} \\
s+3(-p+4 q-1) & =\|2\|=2 a_{1}+(-2 p+4 q) a_{2}+2 a_{3} \\
s+4(-p+3 q-1) & =\|3\|=a_{1}+(-3 p+4 q) a_{2}+3 a_{3} \\
s & =\|\infty\|=a_{1}-p a_{2}+a_{3}
\end{aligned}
$$

which is equivalent in matrix form to

$$
\underbrace{\left(\begin{array}{cccc}
3 & -p+4 q & 1 & -1 \\
2 & -2 p+4 q & 2 & -1 \\
1 & -3 p+4 q & 3 & -1 \\
1 & -p & 1 & -1
\end{array}\right)}_{A}\left(\begin{array}{c}
a_{1} \\
a_{2} \\
a_{3} \\
s
\end{array}\right)=\left(\begin{array}{c}
2(-p-1)+12 q \\
3(-p-1)+12 q \\
4(-p-1)+12 q \\
0
\end{array}\right)
$$

Since the second row of $A$ is equal to one half of the sum of the first and third rows, the rank of $A$ is seen to be three, and the system is therefore underdetermined. We therefore need more information to determine the solution.

Using the bound from table 5] we have $s \leq-3 p+4 q-3$. In other words, we have

$$
s=-3 p+4 q-3-z,
$$

where $z=z(p, q) \geq 0$ is some positive, integer-valued function in $p$ and $q$.

Using this expression for $s$, we can formally solve the system (83) to obtain the following solution in terms of $z$ :

$$
\left(\begin{array}{c}
a_{1} \\
a_{2} \\
a_{3} \\
s
\end{array}\right)=\left(\begin{array}{c}
-p+2 q-1-\frac{z}{2} \\
2+\frac{z}{4 q} \\
2 q-2+z \frac{p-2 q}{4 q} \\
-3 p+4 q-3-z
\end{array}\right)
$$

We can now use the fact that $a_{1}, a_{2}, a_{3}$ and $s$ all are even, non-negative integers: The expression for $a_{2}$ then implies that $z$ is divisible by $8 q$, i.e. $z=8 q z^{\prime}$ for some non-negative integer-valued $z^{\prime}=z^{\prime}(p, q)$. Using this in the expression for $a_{3}$ then shows that $2 q-2+$ $2 z^{\prime}(p-2 q) \geq 0$, which implies that

$$
z^{\prime}(p-2 q) \geq 1-q
$$


Since $p<0$ and $q>0$ implies $p-2 q<0$, we then get

$$
z^{\prime} \leq \frac{1-q}{p-2 q}=\frac{q-1}{2(q+|p| / 2)}<1 .
$$

But since $z^{\prime} \in \mathbb{Z}_{+}$, we must conclude that in fact $z^{\prime}=z=0$.

This proves Theorem 1 and Corollary 4 for $p / q<0$.

6.2. Assuming $p / q \in(0,2), p$ odd. In this case, we obtain the following distances between the relevant slopes:

\begin{tabular}{c|ccccccc}
$\Delta$ & $\beta_{1}=4$ & $\beta_{2}=\frac{2 p+4 q}{p}$ & $\beta_{3}=0$ & 1 & 2 & 3 & $\mu=\infty$ \\
\hline$\beta_{1}$ & 0 & $-2 p+4 q$ & 4 & 3 & 2 & 1 & 1 \\
$\beta_{2}$ & & 0 & $2 p+4 q$ & $p+4 q$ & $4 q$ & $-p+4 q$ & $p$ \\
$\beta_{3}$ & & & 0 & 1 & 2 & 3 & 1
\end{tabular}

TABLE 11. Distances between slopes in $W_{p / q}$ for $p / q \in(0,2), p$ odd

The corresponding linear system is in this case given by the equations:

$$
\begin{aligned}
s+2(-p+6 q-1) & =\|1\|=3 a_{1}+(p+4 q) a_{2}+a_{3} \\
s+3(-p+4 q-1) & =\|2\|=2 a_{1}+4 q a_{2}+2 a_{3} \\
s+4(-p+3 q-1) & =\|3\|=a_{1}+(-p+4 q) a_{2}+3 a_{3} \\
s & =\|\infty\|=a_{1}+p a_{2}+a_{3}
\end{aligned}
$$

Using the upper bound $s \leq p+4 q-3$ from table 5 we can assume

$$
s=p+4 q-3-z,
$$

where $z=z(p, q) \geq 0$ is some positive, integer-valued function in $p$ and $q$.

Using this expression for $s$, we can formally solve the system (88) to obtain the following solution in terms of $z$ :

$$
\left(\begin{array}{c}
a_{1} \\
a_{2} \\
a_{3} \\
s
\end{array}\right)=\left(\begin{array}{c}
-p+2 q-1-\frac{q z}{2 q-p} \\
2+\frac{z}{2(2 q-p)} \\
2 q-2-\frac{z}{2} \\
p+4 q-3-z
\end{array}\right)
$$

Now recall that $a_{1}, a_{2}, a_{3}$ and $s$ all are even, non-negative integers. The expression for $a_{2}$ then implies that $z$ is divisible by $4(2 q-p)$, i.e. $z=4(2 q-p) z^{\prime}$ for some nonnegative integer-valued $z^{\prime}=z^{\prime}(p, q)$. Using this in the expression for $a_{1}$ then shows that $-p+2 q-1-4 q z^{\prime} \geq 0$, which implies that

$$
z^{\prime} \leq \frac{-p+2 q-1}{4 q}=1-\frac{p+1+2 q}{4 q}<1,
$$

so in fact $z^{\prime}=z=0$, proving Theorem 1 and Corollary 4 in the case $p / q \in(0,2)$.

6.3. Assuming $p / q \in(2,3) \cup(3,4), p$ odd. In this case, we obtain the following distances between the relevant slopes:

\begin{tabular}{c|ccccccc}
$\Delta$ & $\beta_{1}=4$ & $\beta_{2}=\frac{-p+6 q}{q}$ & $\beta_{3}=0$ & 1 & 2 & 3 & $\mu=\infty$ \\
\hline$\beta_{1}$ & 0 & $p-2 q$ & 4 & 3 & 2 & 1 & 1 \\
$\beta_{2}$ & & 0 & $-p+6 q$ & $-p+5 q$ & $-p+4 q$ & $|p-3 q|$ & $q$ \\
$\beta_{3}$ & & & 0 & 1 & 2 & 3 & 1
\end{tabular}

TABLE 12. Distances between slopes in $W_{p / q}$ for $p / q \in(2,4), p$ odd 
In this case, the corresponding linear system is:

$$
\begin{aligned}
s+2(-p+6 q-1) & =\|1\|=3 a_{1}+(-p+5 q) a_{2}+a_{3} \\
s+3(-p+4 q-1) & =\|2\|=2 a_{1}+(-p+4 q) a_{2}+2 a_{3} \\
s+4(|p-3 q|-1) & =\|3\|=a_{1}+|p-3 q| a_{2}+3 a_{3} \\
s & =\|\infty\|=a_{1}+q a_{2}+a_{3}
\end{aligned}
$$

If $p / q \in(3,4)$, this system already has rank 4 , and we can determine the unique solution without further assumptions:

$$
\left(\begin{array}{c}
a_{1} \\
a_{2} \\
a_{3} \\
s
\end{array}\right)=\left(\begin{array}{c}
p-2 q-1 \\
4 \\
2 q-2 \\
p+4 q-3
\end{array}\right)
$$

This proves Theorem 1 and Corollary 4 for $p / q \in(3,4)$.

If on the other hand $p / q \in(2,3)$, the system has only rank 3 . Furthermore, using the upper bound for the minimal seminorm does not yet determine the system in this case.

But now recall from Lemma 10 (4) that $\operatorname{res}_{p, q} \doteq \mathrm{res}_{-p+4 q, q}$. Furthermore, $2<p / q<3$ if and only if $1<-p / q+4<2$, and so the simplicity of the non-trivial roots of $\operatorname{res}_{p, q}$ when $p / q \in(2,3)$ follows from the simplicity of the non-trivial roots of $\operatorname{res}_{-p+4 q, q}$ when $\frac{-p+4 q}{q} \in(1,2)$, which we have already proven in section 6.2 We can therefore already assume the validity of Corollary 4 for $p / q \in(2,3)$ and use that $s$ actually attains the upper bound from table 5] i.e. $s=p+4 q-3$. Solving the linear system with this information, we then obtain the same solution as given in equation (93), completing the proof of Theorem 1 in this case.

6.4. Assuming $p / q \in(4,6) \cup(6, \infty), p$ odd. In this case, we obtain the following distances between the relevant slopes:

\begin{tabular}{c|ccccccc}
$\Delta$ & $\beta_{1}=4$ & $\beta_{2}=\frac{4 q}{p-2 q}$ & $\beta_{3}=0$ & 1 & 2 & 3 & $\mu=\infty$ \\
\hline$\beta_{1}$ & 0 & $4 p-12 q$ & 4 & 3 & 2 & 1 & 1 \\
$\beta_{2}$ & & 0 & $4 q$ & $|p-6 q|$ & $2 p-8 q$ & $3 p-10 q$ & $p-2 q$ \\
$\beta_{3}$ & & & 0 & 1 & 2 & 3 & 1
\end{tabular}

TABLE 13. Distances between slopes in $W_{p / q}$ for $p / q \in(4, \infty), p$ odd

Similar to the previous cases, we obtain the corresponding linear system as:

$$
\begin{aligned}
s+2(|p-6 q|-1) & =\|1\|=3 a_{1}+|p-6 q| a_{2}+a_{3} \\
s+3(p-4 q-1) & =\|2\|=2 a_{1}+(2 p-8 q) a_{2}+2 a_{3} \\
s+4(p-3 q-1) & =\|3\|=a_{1}+(3 p-10 q) a_{2}+3 a_{3} \\
s & =\|\infty\|=a_{1}+(p-2 q) a_{2}+a_{3}
\end{aligned}
$$

If $p / q \in(4,6)$, this system has rank 4 , and we can determine the unique solution without further assumptions:

$$
\left(\begin{array}{c}
a_{1} \\
a_{2} \\
a_{3} \\
s
\end{array}\right)=\left(\begin{array}{c}
p-2 q-1 \\
2 \\
2 q-2 \\
3 p-4 q-3
\end{array}\right)
$$

This then proves Theorem 1 and Corollary 4 for $p / q \in(4,6)$.

If on the other hand $p / q \in(6, \infty)$, the system has only rank 3 . Furthermore, using the upper bound for the minimal seminorm does not yet determine the system in this case. 
But the same argument as used in the previous subsection holds here: By Lemma 10 (4), we have that $\operatorname{res}_{p, q} \doteq \operatorname{res}_{-p+4 q, q}$. Furthermore, $p / q>6$ if and only if $-p / q+4<-2$, and so the simplicity of the non-trivial roots of $\operatorname{res}_{p, q}$ when $p / q>6$ follows from the simplicity of the non-trivial roots of $\operatorname{res}_{-p+4 q, q}$ when $\frac{-p+4 q}{q}<-2$, which we have already proven in section 6.1. We can therefore already assume Corollary 4 for $p / q \in(6, \infty)$ and use that $s$ actually attains the upper bound from table 5] i.e. $s=3 p-4 q-3$. Solving the linear system with this information, we then obtain the same solution as given in equation (95), completing the proof of Theorem 1

\section{References}

[BAB01] L. Ben Abdelghani and S. Boyer, A calculation of the Culler-Shalen seminorms associated to small Seifert Dehn fillings, Proc. London Math. Soc. (3) 83 (2001), no. 1, 235-256. MR 1829566 (pages 25 27 28).

[BGZ01] S. Boyer, C. McA. Gordon, and X. Zhang, Dehn fillings of large hyperbolic 3-manifolds, J. Differential Geom. 58 (2001), no. 2, 263-308. MR 1913944 (page 2).

[BMZ97] S. Boyer, T. Mattman, and X. Zhang, The fundamental polygons of twist knots and the $(-2,3,7)$ pretzel knot, KNOTS '96 (Tokyo), World Sci. Publishing, River Edge, NJ, 1997, pp. 159-172. MR 1664959 (page 2).

[Boy02] S. Boyer, On the local structure of $\mathrm{SL}(2, \mathbb{C})$-character varieties at reducible characters, Topology Appl. 121 (2002), no. 3, 383-413. MR 1909000 (pages 16, 24).

[Bro82] K. S. Brown, Cohomology of groups, Graduate Texts in Mathematics, vol. 87, SpringerVerlag, New York, 1982. MR 672956 (page 20).

[BZ96] S. Boyer and X. Zhang, Finite Dehn surgery on knots, J. Amer. Math. Soc. 9 (1996), no. 4, 1005-1050. MR 1333293 (pages 3 6 30).

[BZ98] - On Culler-Shalen seminorms and Dehn filling, Ann. of Math. (2) 148 (1998), no. 3, 737-801. MR 1670053 (pages 5, 17).

[BZ03] G. Burde and H. Zieschang, Knots, second ed., de Gruyter Studies in Mathematics, vol. 5, Walter de Gruyter \& Co., Berlin, 2003. MR 1959408 (page 18).

$\left[\mathrm{CCG}^{+94]}\right.$ D. Cooper, M. Culler, H. Gillet, D. D. Long, and P. B. Shalen, Plane curves associated to character varieties of 3-manifolds, Invent. Math. 118 (1994), no. 1, 47-84. MR 1288467 (page 5).

[CGLS87] M. Culler, C. McA. Gordon, J. Luecke, and P. B. Shalen, Dehn surgery on knots, Ann. of Math. (2) 125 (1987), no. 2, 237-300. MR 881270 (pages 5 16).

[CLO97] D. Cox, J. Little, and D. O'Shea, Ideals, varieties, and algorithms, second ed., Undergraduate Texts in Mathematics, Springer-Verlag, New York, 1997. MR 1417938 (pages 11, 19).

[CS83] M. Culler and P. B. Shalen, Varieties of group representations and splittings of 3manifolds, Ann. of Math. (2) 117 (1983), no. 1, 109-146. MR 683804 (page 5).

[DT03] N. M. Dunfield and W. P. Thurston, The virtual Haken conjecture: experiments and examples, Geom. Topol. 7 (2003), 399-441 (electronic). MR 1988291 (page 27).

[FH88] W. Floyd and A. Hatcher, The space of incompressible surfaces in a 2-bridge link complement, Trans. Amer. Math. Soc. 305 (1988), no. 2, 575-599. MR 924770 (page 6).

[Fox53] R. H. Fox, Free differential calculus. I. Derivation in the free group ring, Ann. of Math. (2) 57 (1953), 547-560. MR 0053938 (page [18).

[Gol84] W. M. Goldman, The symplectic nature of fundamental groups of surfaces, Adv. in Math. 54 (1984), no. 2, 200-225. MR 762512 (page 24).

[HMW92] C. D. Hodgson, G. R. Meyerhoff, and J. R. Weeks, Surgeries on the Whitehead link yield geometrically similar manifolds, Topology '90 (Columbus, OH, 1990), Ohio State Univ. Math. Res. Inst. Publ., vol. 1, de Gruyter, Berlin, 1992, pp. 195-206. MR 1184411 (pages 22 18).

[HP05] M. Heusener and J. Porti, Deformations of reducible representations of 3-manifold groups into $\mathrm{PSL}_{2}(\mathbb{C})$, Algebr. Geom. Topol. 5 (2005), 965-997 (electronic). MR 2171800 (pages 16, 18, 19, 20).

[HPSP01] M. Heusener, J. Porti, and E. Suárez Peiró, Deformations of reducible representations of 3-manifold groups into $\mathrm{SL}_{2}(\mathbf{C})$, J. Reine Angew. Math. 530 (2001), 191-227. MR 1807271 (pages 20, 24). 
[HS07] J. Hoste and P. D. Shanahan, Computing boundary slopes of 2-bridge links, Math. Comp. 76 (2007), no. 259, 1521-1545 (electronic). MR 2299787 (page 6).

[Jac80] W. Jaco, Lectures on three-manifold topology, CBMS Regional Conference Series in Mathematics, vol. 43, American Mathematical Society, Providence, R.I., 1980. MR 565450 (page 28).

[Las93] A. E. Lash, Boundary curve space of the Whitehead link complement, Ph.D. thesis, University of California, Santa Barbara, 1993. (page6).

[Map] Maplesoft, a division of Waterloo Maple Inc., Waterloo, Ontario., Maple. (page 22).

[Mat02] T. W. Mattman, The Culler-Shalen seminorms of the $(-2,3, n)$ pretzel knot, J. Knot Theory Ramifications 11 (2002), no. 8, 1251-1289. MR 1949779 (page 6).

[MP06] Bruno Martelli and Carlo Petronio, Dehn filling of the "magic" 3-manifold, Comm. Anal. Geom. 14 (2006), no. 5, 969-1026. MR 2287152 (page27).

[Por97] J. Porti, Torsion de Reidemeister pour les variétés hyperboliques, Mem. Amer. Math. Soc. 128 (1997), no. 612, x+139. MR 1396960 (page 20).

[Ril72] R. Riley, Parabolic representations of knot groups. I, Proc. London Math. Soc. (3) 24 (1972), 217-242. MR 0300267 (pages 3 8).

[Ril75] _ Parabolic representations of knot groups. II, Proc. London Math. Soc. (3) 31 (1975), no. 4, 495-512. MR 0413078 (page 8).

[Sha94] I. R. Shafarevich, Basic algebraic geometry. 1. Varieties in projective space, second ed., Springer-Verlag, Berlin, 1994. MR 1328833 (page 5).

[Sha02] P. B. Shalen, Representations of 3-manifold groups, Handbook of geometric topology, North-Holland, Amsterdam, 2002, pp. 955-1044. MR 1886685 (page 16).

[Sze75] G. Szegö, Orthogonal polynomials, fourth ed., AMS Colloquium Publications, Vol. XXIII, American Mathematical Society, Providence, R.I., 1975. MR 0372517 (pages 11, 13).

[Thu79] W. P. Thurston, Geometry and Topology of Three-Manifolds. Princeton lecture notes, 1979. (page 2).

[Til02] S. Tillmann, On character varieties: Surfaces associated to mutation \& deformation of hyperbolic 3-manifolds, Ph.D. thesis, University of Melbourne, August 2002. (pages 8,9 (10).

[Til05] Boundary slopes and the logarithmic limit set, Topology 44 (2005), no. 1, 203-216. MR 2104008 (pages 3 9).

[Til19] - Tropical varieties associated to ideal triangulations: The Whitehead link complement, arXiv e-prints (2019), arXiv:1911.04619. (pages 8 , 9 10).

[Wad94] M. Wada, Twisted Alexander polynomial for finitely presentable groups, Topology 33 (1994), no. 2, 241-256. MR 1273784 (page 18).

[Wei64] A. Weil, Remarks on the cohomology of groups, Ann. of Math. (2) 80 (1964), 149-157. MR 0169956 (page 20).

Département de mathématiques, 201, Avenue du Président-Kennedy, Montréal (Québec), CANADA H2X 3 Y7

Current address: Department of Mathematics, Champlain College St-Lambert, 900 Riverside

Drive, St-Lambert (Québec), Canada J4P 3P2

E-mail address: gindurskis@crcmail.net 